\title{
ORDENAMIENTO Y CARACTERIZACIÓN FAUNÍSTICA DEL CRETACICO SUPERIOR DEL GRUPO CHUBUT, CUENCA DEL GOLFO SAN JORGE, ARGENTINA
}

\author{
GABRIEL A. CASAL, RUBÉN D. MARTÍNEZ, MARCELO LUNA \\ Laboratorio de Paleovertebrados, Departamento de Geología, Facultad de Ciencias Naturales,Universidad Nacional de la \\ Patagonia San Juan Bosco, Ruta provincial n¹, km 4, C.P. 9000 Comodoro Rivadavia, Chubut, Argentina. \\ paleogac@yahoo.com.ar,rudaframartinez@gmail.com,paleoambiental@yahoo.com \\ LUCIO M. IBIRICU \\ Instituto Patagónico de Geología y Paleontología (IPGP-CONICET), Boulevard Almirante Brown 2915, 9120 \\ Puerto Madryn, Chubut, Argentina. ibiricu@cenpat-conicet.gob.ar
}

\begin{abstract}
FAUNISTIC ARRANGEMENT OF THE UPPER CRETACEOUS OF CHUBUT GROUP, GULF SAN JORGE BASIN, ARGENTINA. The Chubut Group, central Patagonia, Argentina, is characterized by a lacustrine and fluvial-lacustrine system with variable participation of volcanic ash. This group includes the Bajo Barreal Formation (Cenomanian-Turonian) and a recently nested new lithostratigraphic unit, the Lago Colhué Huapi Formation (Coniacian-Maastrichtian). The Lago Colhué Huapi Formation overlies the Bajo Barreal Formation. These sedimentary units preserve a rich and diverse vertebrate fossil record including, among others, representatives of Crocodylomorpha, Testudines, Pterosauria and abundant Dinosauria. Nevertheless, the stratigraphic position of several of its taxa has been historically controversial. The unclear stratigraphic provenance of these taxa difficults the correct interpretation of the relationships with other Patagonian and South American basins. In this context, we present a detailed stratigraphic study to clarify the position of the vertebrate fossils of both Late Cretaceous formations. We also discuss the implications of this faunistic arrangement in terms of vertebrate evolution and paleobiogeography. Finally, this study broadens our knowledge on the fossil fauna of these units and therefore the vertebrate assemblages of central Patagonia.
\end{abstract}

Key words: Chubut Group, Bajo Barreal and Lago Colhué Huapi formations, Late Cretaceous, vertebrate assemblages.

RESUMO - O Grupo Chubut, Patagônia central, Argentina, é caracterizado por um sistema lacustre e flúvio-lacustre, com uma participação variável de cinza vulcânica. Este grupo inclui a Formação Bajo Barrel (Cenomaniano-Turoniano) e uma nova unidade litoestratigráfica recentemente depositada, a Formação Lago Colhué Huapi (Coniaciano-Maastrichtiano). A Formação Lago Colhué Huapi se sobrepõe à Bajo Barreal. Estas unidades sedimentares contêm um rico e diversificado registro fóssil de vertebrados que inclui, entre outros, representantes de Crocodylomorpha, Testudines, Pterosauria e abundantes, Dinosauria. Entretanto, as posições estratigráficas de muitos destes táxons são historicamente controversas. A origem estratigráfica pouco clara desses táxons, dificulta a interpretação correta das relações de seus fósseis com a fauna de outras bacias patagônicas e sul-americanas. Sendo assim, dentro deste contexto, nós apresentamos um posicionamento estratigráfico detalhado dos vertebrados fósseis das duas formações do Cretácio Superior e discutimos as implicações destes arranjos faunísticos em termos de evolução dos vertebrados e paleobiogeografia. Por fim, o presente estudo amplia nosso conhecimento sobre a fauna fóssil destas unidades e também das assembleias de vertebrados da Patagônia central.

Palavras-chave: Grupo Chubut, formações Bajo Barreal e Lago Colhué Huapi, Cretáceo Superior, assembleias de vertebrados.

\section{INTRODUCCIÓN}

La Cuenca del Golfo San Jorge posee un basamento integrado por rocas metamórficas del Paleozoico Superior, calizas, tobas y vulcanitas ácidas jurásicas del Grupo Bahía Laura y el Grupo Lonco Trapial (Figari et al., 2002). En discordancia se depositó un relleno sedimentario continental que constituye el Grupo Chubut (Lesta \& Ferello, 1972), caracterizado por sistemas lacustres y fluvio-lacustres con variable participación de ceniza volcánica (Umazano et al., 2008, 2012). La sedimentación del grupo comprende gran parte del Cretácico Superior e incluía a las formaciones Pozo D-129, Matasiete, Castillo, Bajo Barreal y Laguna Palacios de acuerdo con Lesta \& Ferello (1972).

Recientemente, Casal et al. (2015a) propusieron modificar este clásico esquema del Grupo Chubut con la incorporación de la Formación Lago Colhué Huapi (Coniaciano-Maastrichtiano), extendiendo cronológicamente al Grupo Chubut desde el Aptiano hasta el Maastrichtiano (Figura 1), alcanzando con esta nueva unidad el registro más moderno del Cretácico Superior. 
Algunas de estas unidades mencionadas, no solo son productoras de hidrocarburos, sino también portadoras de un alto contenido fosilífero. En particular, la Formación Bajo Barreal (Cenomaniano-Turoniano) se destaca por su riqueza y diversidad faunística de vertebrados fósiles, en especial la parte alta de su Miembro Inferior. Esta unidad sedimentaria, aflora en el sector de la faja plegada de San Bernardo, y su fauna procedente fundamentalmente de las localidades de Estancia Ocho Hermanos y Cañadón Las Horquetas (Figura 2). Luego de casi cuatro décadas de exploraciones y estudios ha brindado significativa información desde el punto de vista morfológico, sistemático, evolutivo (Powell et al., 1989; Martínez, 1998a,b; 2004a; Martínez \& Novas, 2006; Casal et al., 2009; Casal \& Ibiricu, 2010; Ibiricu et al., 2012a,b; Ibiricu et al., 2013a,b, entre otros), paleogeográfico, paleoecológico (Lamanna et al., 2002; Ibiricu et al., 2012a; Ibiricu et al., 2014) y tafonómico (Rodríguez, 1993; Casal et al., 2013), transformándose en una unidad cretácica de relevancia mundial.

En contraste, las secuencias sedimentarias cretácicas de la zona del lago Colhué Huapi y nacientes del río Chico (Figura 2), han aportado menos información, en parte debido a la menor prospección de sus niveles. No obstante, el registro fósil de esta región se ha incrementado en los últimos años a partir de exploraciones y estudios sistemáticos realizados desde el Laboratorio de Paleovertebrados de la Universidad Nacional de la Patagonia San Juan Bosco, de Comodoro Rivadavia (Lamanna et al., 2003; Luna et al., 2003; Casal et al., 2006; Casal et al., 2007; Ibiricu et al., 2010; Casal et al., 2010; Casal et al., 2015b). Pese a esto, aspectos geológicos y paleontológicos de esta zona han sido históricamente confusos, resultando en muchos casos conflictiva la ubicación estratigráfica y la edad de sus fósiles.

Basados en la reciente definición de la nueva Formación Lago Colhué Huapi (Casal et al., 2015a) para el Cretácico
Superior de la Cuenca del Golfo San Jorge, en este trabajo se pretende escindir y ordenar estratigráfica, y cronológicamente el contenido paleontológico del intervalo estratigráfico más alto del Grupo Chubut (formaciones Bajo Barreal y Lago Colhué Huapi). Dicho ordenamiento y su consecuente caracterización faunística, es de suma importancia para el entendimiento de la evolución de la fauna cretácica de la cuenca mencionada y sus vinculaciones con otras cuencas patagónicas y gondwánicas.

Abreviaturas institucionales. FMNH, Field Museum of Natural History, Chicago, Illinois, Estados Unidos; DNM, Dirección Nacional de Minas, Provincia de San Juan, Argentina; MACN-CH, Museo Argentino de Ciencias Naturales, Colección Chubut, Buenos Aires, Argentina; MDTPv, Museo Desiderio Torres, Paleontología de vertebrados, Sarmiento, Provincia del Chubut, Argentina; MLP-V, Museo de La Plata, Vertebrados, Provincia de Buenos Aires, Argentina; UNPSJB-Pv, Universidad Nacional de la Patagonia San Juan Bosco, Paleontología de vertebrados, Comodoro Rivadavia, Provincia del Chubut, Argentina; MPM, Museo Padre Manuel Molina, Río Gallegos, Provincia de Santa Cruz, Argentina.

\section{MATERIAL Y MÉTODOS}

El registro paleontológico fue analizado mediante una revisión bibliográfica de los hallazgos realizados en la región del lago Colhué Huapi, nacientes del río Chico y codo del río Senguerr y Estancia "Ocho Hermanos" y Cañadón "Las Horquetas". Asimismo, este registro fue ampliado a partir de distintas prospecciones de dichos afloramientos realizadas sistemáticamente por el Laboratorio de Paleovertebrados de la UNPSJB.

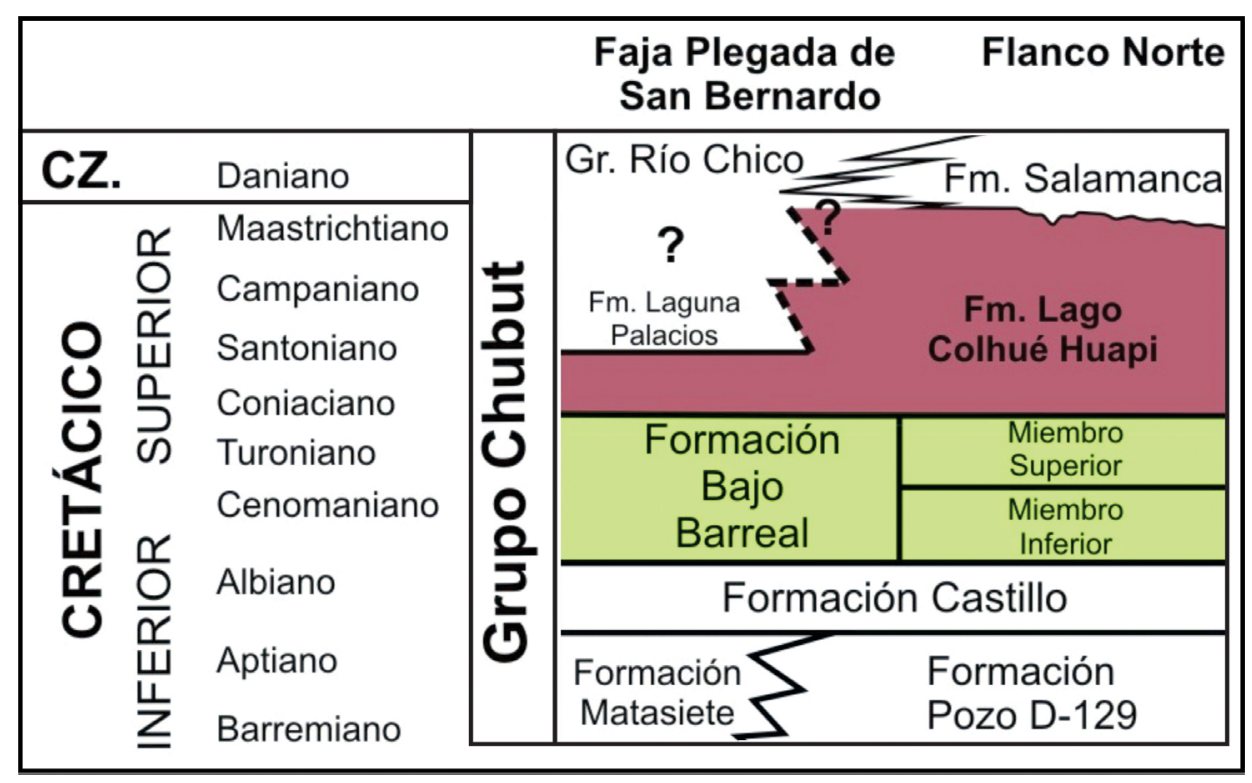

Figura 1. Cuadro estratigráfico del Grupo Chubut (modificado de Casal et al., 2015a).

Figure 1. Stratigraphic column of Chubut Group (modified from Casal et al., 2015a). 


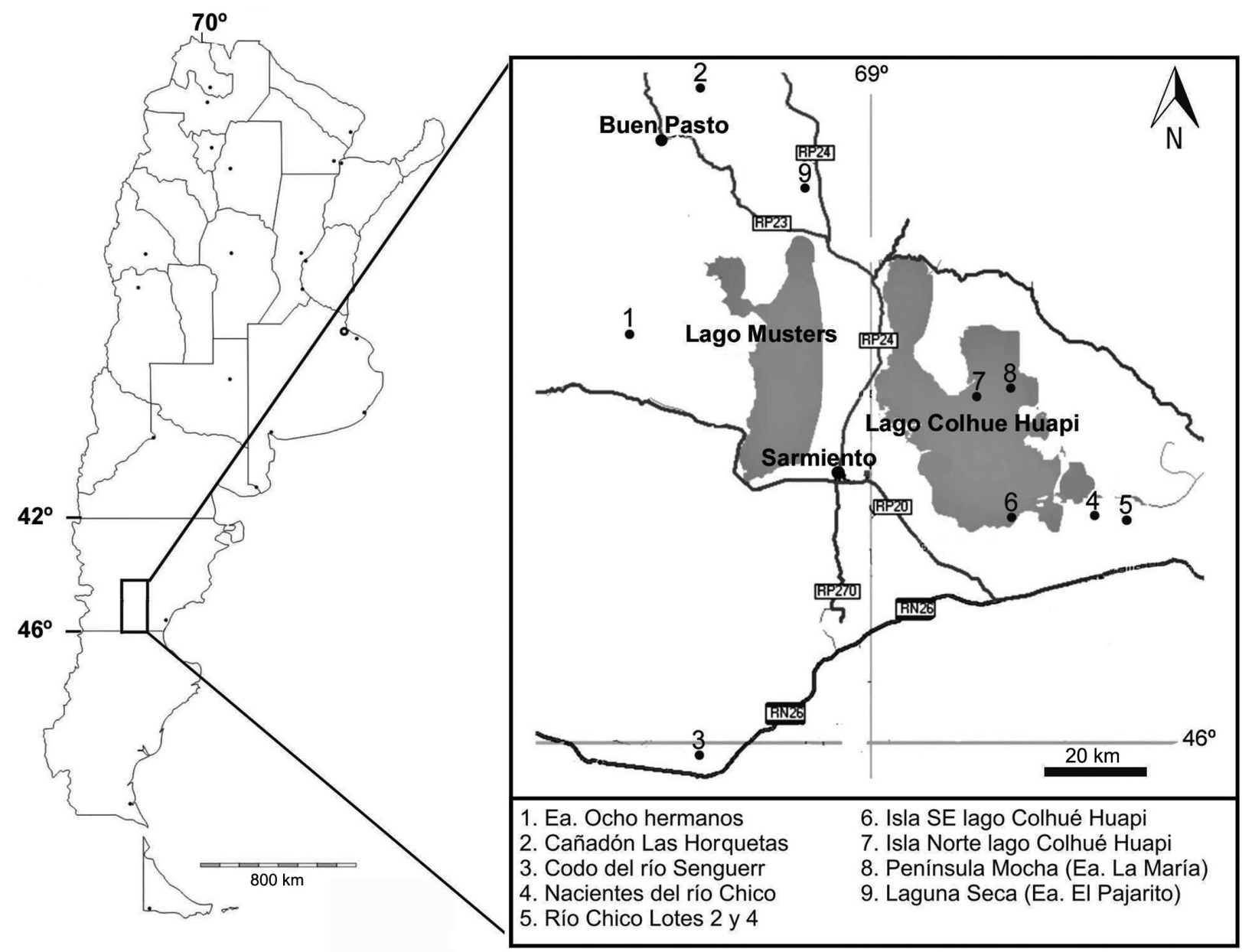

Figura 2. Mapa de ubicación geográfica de los sitios de procedencia de los restos fósiles.

Figure 2. Map showing locations of sites where the fossils were discovered.

\section{RESULTADOS}

\section{Síntesis estratigráfica de la Formación Bajo Barreal}

La Formación Bajo Barreal fue definida por Teruggi \& Rossetto (1963) y equivale a las denominaciones de Chubutiano Superior (Roll, 1938), Tobas grises (Feruglio, 1949), Serie del Bajo Barreal (Ferello \& Tealdi, 1950) y Sección IV (Tobas Grises) del Chubutense (Vilela, 1971). La sedimentación de la unidad se corresponde con depósitos de sistemas fluviales fuertemente influenciados por el vulcanismo explosivo contemporáneo (Umazano et al., 2008). Sciutto (1981) y Hechem et al. (1990) reconocieron dos miembros en la unidad. El Miembro Inferior se presenta conformando bancos medianos a gruesos y bien estratificados de areniscas verdes y grises, con estructuras entrecruzadas producto del depósito de canales de baja sinuosidad y diseño entrelazado (Rodríguez, 1992, 1993; Figari et al., 1999).

Los canales se asocian con una reducida planicie de inundación integrada por fangolitas grises que se depositaron en ambientes de bajo gradiente paleotopográfico, probablemente en condiciones de planicie de inundación mal drenadas. Frecuentemente se intercalan bancos tobáceos
(Sciutto, 1981; Paredes, 2009). Sin embargo, el registro de depósitos piroclásticos primarios suele ser escaso porque fueron reelaborados por los cursos fluviales (Umazano et al., 2008), o bien se encuentran edafizados en diferente grado, constituyendo paleosuelos. Rodríguez (1993) analizó estos depósitos en la estancia Ocho Hermanos, en el flanco oriental de la sierra San Bernardo, sugiriendo que la unidad fue depositada por un sistema de corrientes efímeras, confinadas e inconfinadas limitadas por planicies fangosas del tipo barreales. Lateralmente, se asocian depósitos de desbordamiento que representan el principal mecanismo de agradación de la planicie de inundación quedando subordinadas las lagunas poco profundas (Umazano et al., 2008). El sector más alto del Miembro Inferior presenta una importante concentración de restos fósiles de vertebrados localizados en estos depósitos de desbordamiento evidenciando condiciones tafonómicas y sedimentarias apropiadas para su preservación (Rodríguez, 1993; Casal et al., 2013).

El Miembro Superior presenta una topografía de lomadas redondeadas, a veces del tipo bad lands. Está integrado por bancos de fangolitas grises a castañas (Figuras 3A-B) que predominan marcadamente sobre las areniscas de canales 
que forman lentes de poca potencia. Fue interpretado como una planicie de inundación limo-arcillosa por Sciutto (1981), mientras que para Umazano et al. (2008) su depositación se produjo durante condiciones eólicas inter-eruptivas. En este miembro, la presencia de restos de vertebrados fósiles es escasa, destacándose, al momento, la presencia del titanosaurio Drusilasaura deseadensis Navarrete, Casal \& Martínez, 2011.

Finalmente, los frecuentes depósitos piroclásticos y las facies de planicie de inundación se encuentran edafizados en diferente grado, constituyendo paleosuelos que indican el predominio de condiciones subacuaticas e inundadas (Bellosi et al., 2000).

\section{Edad de la Formación Bajo Barreal}

La edad asignada a esta unidad litoestratigráfica ha variado según diversos autores: Bonaparte \& Gasparini (1979) la asignaron al Senoniano en base a la fauna de vertebrados fósiles registrada. Powell et al. (1989) la ubican en el intervalo Aptiano-Cenomaniano con posibilidades de alcanzar el Senoniano temprano. Posteriormente, y a partir del estudio de esporas y granos de polen en rocas de subsuelo equivalentes al Miembro Inferior de la Formación Bajo Barreal (Formación Cañadón Seco, Miembro Caleta Olivia), Archangelsky et al. (1994) la asignan al Albiano tardío-Cenomaniano. Chelotti \& Homove (1998) la ubican en el intervalo Turoniano-Maastrichtiano. En base a dataciones radimétricas (Ar/Ar) sobre tobas, Bridge et al. (2000) obtuvieron valores de 94,2 $\pm 0,63 \mathrm{Ma}$. para el Miembro Inferior de la Formación Bajo Barreal y 91 $\pm 0,49$ Ma. para el Miembro Superior, lo que permite ubicar la unidad en el Cenomaniano medio-Turoniano tardío. En base a la fauna dinosauriana, Lamanna et al. (2002) la asignan al Cenomaniano tardío-Turoniano temprano. Recientemente, Suárez et al. (2014) realizaron dataciones (U/Pb SHRIMP) en muestras procedentes del Miembro Inferior, y obtuvieron valores de 99,3 $\pm 0,7$ ubicando a esta unidad en el Cenomaniano temprano. Con esta información disponible y en base a los índices de similaridad faunística determinados por Canale et al. (2011) entre esta unidad y las unidades del Subgrupo Río Limay (formaciones Candeleros y Huincul) de la Cuenca Neuquina, en este trabajo se apoya una edad Cenomaniano temprano-Turoniano tardío para la Formación Bajo Barreal.

\section{Paleoclima de la Formación Bajo Barreal}

La abundancia de paleosuelos en la unidad sugiere la presencia de un clima cálido y húmedo apropiado para el desarrollo de paleosuelos, a diferencia de los climas áridos que inhiben estos procesos (Retallack, 1988). Al respecto, un estudio sobre el paleoclima de la Formación Bajo Barreal realizado por Pujana et al. (2007), a partir de maderas fósiles asignadas a Agathoxylon sp. (Araucariacea), indican la ausencia de estacionalidad sobre la base de anillos de crecimiento poco marcados. Este carácter generalmente se vincula a climas templados cálidos a tropicales. Además, Archangelsky et al. (1994) y Barreda \& Archangelsky (2006) también determinan la presencia de un clima cálido y húmedo para el Cenomaniano de la Cuenca del Golfo San Jorge, a partir de estudios palinológicos.
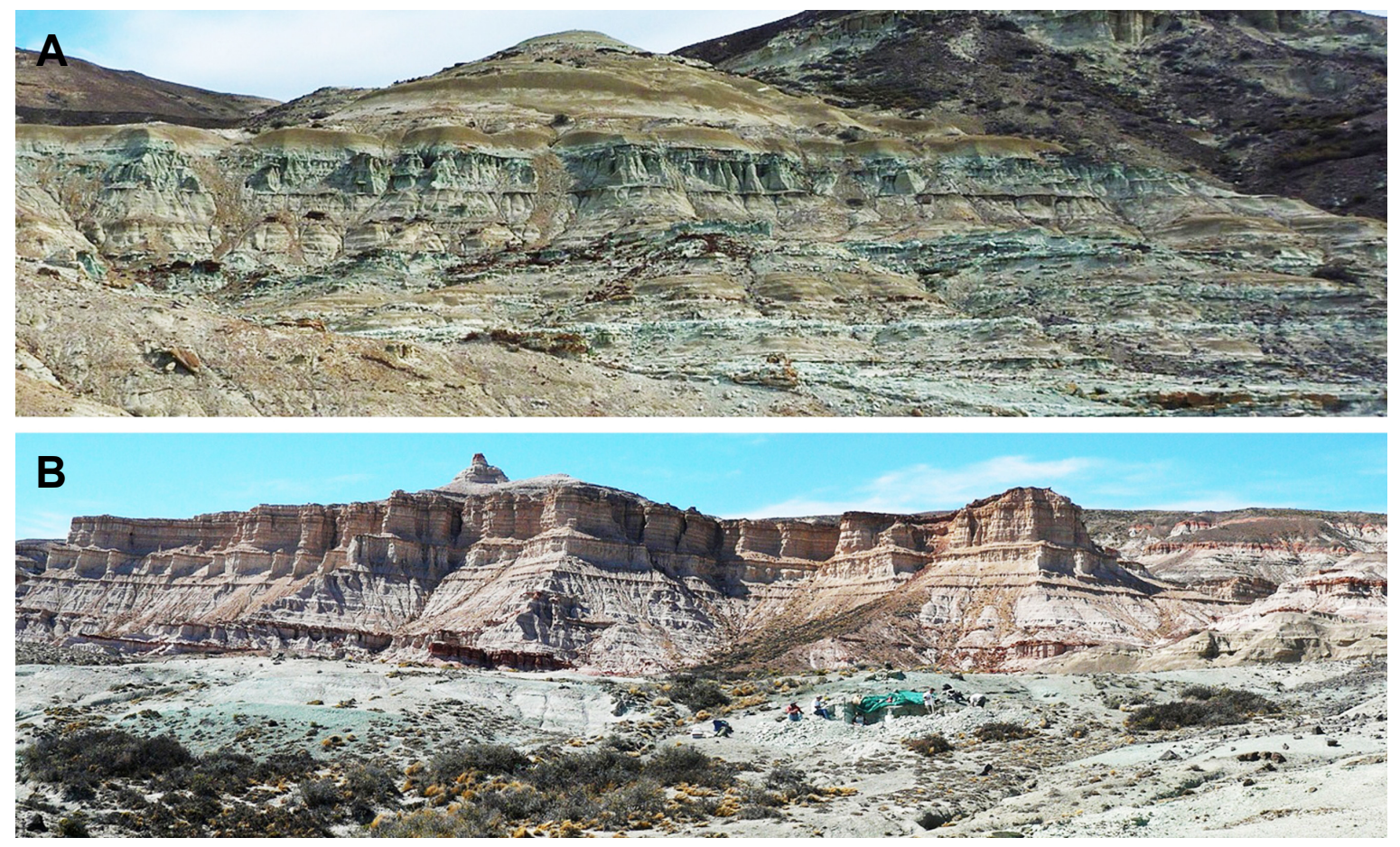

Figura 3. Afloramientos de la Formación Bajo Barreal donde se observan las típicas areniscas verdes. A, en Estancia Ocho Hermanos; B, en cañadón Las Horquetas, Sur de la Provincia del Chubut, Argentina.

Figure 3. Outcrops of the Bajo Barreal Formation with the characteristic green sandstones. A, in Ea. Ocho Hermanos; B, in cañadón Las Horquetas, South of Chubut Province, Argentina. 


\section{Paleontología de la Formación Bajo Barreal}

Los principales sitios de procedencia de los vertebrados fósiles de la Cuenca del Golfo San Jorge corresponden a la Estancia Ocho Hermanos, en la Sierra San Bernardo (Figuras 2, 3A) y al cañadón Las Horquetas, en la Estancia Laguna Palacios, Sierra Nevada, próximo al pueblo Buen Pasto (Figuras 2,3B). En ambos sitios, se han recuperado numerosos restos de diversos taxones (Tabla 1) y que se sintetizan a continuación.

Sauropoda. El maxilar izquierdo incompleto MACN A-IOR63 correspondiente a un saurópodo, del que se preservaron siete alvéolos, un diente funcional y fragmentos de coronas, fue hallado por Carlos Ameghino y nominado por Huene (1929) como Campylodon ameghinoi. Su procedencia geográfica es el flanco Oeste de la sierra San Bernardo sin mayores precisiones (Huene, 1929; Powell, 2003) y su asignación estratigráfica es el Grupo Chubut desconociéndose a que formación específicamente pertenece (Powell, 2003).

Por otra parte, Powell (1990) describió al saurópodo Epachthosaurus sciuttoi a partir de una única vértebra dorsal (MACN-CH 1317) hallada en Estancia Ocho Hermanos. Otros materiales del mismo taxón fueron descubiertos en areniscas muy cementadas y consolidadas que impidieron su extracción y obligaron a la confección de un molde de plástico en el campo (MACN-CH 18689; Powell, 1990). Martínez et al. $(1990,2004 a)$ dieron a conocer nuevos restos articulados y bien preservados de un esqueleto que solo carecía de cráneo, cuello y vértebras dorsales anteriores, referidos también a Epachthosaurus sciuttoi (UNPSJB-Pv 920). Posteriormente, Casal \& Ibiricu (2010) describieron nuevos materiales (UNPSJB-Pv 1006) asignados al mismo género y destacan la abundancia de este taxón en el yacimiento Estancia Ocho Hermanos, y su ausencia hasta el momento en otros sitios de la misma unidad. Por lo tanto, materiales asignables a epactosaurinos constituyen el registro más abundante de saurópodos para la Formación Bajo Barreal, condicionado posiblemente por factores ecológicos (Casal \& Ibiricu, 2010). Por otra parte, Epachthosaurus es considerado el taxón más basal dentro de Lithostrotia con vértebras procélicas a lo largo de toda la cola (Salgado \& Martínez, 1993; Salgado et al., 1997a; Martínez et al., 2004a).

Ibiricu et al. (2011) describieron dos conjunto de vértebras dorsales (UNPSJB-Pv 1010 y 1010/1; UNPSJB-Pv 581 y 582) e identificadas como pertenecientes a titanosaurios basales noepactosaurinos, lo cual diversifica el registro de Titanosauria aunque siempre representado por taxones evolutivamente basales. Asimismo, en estos afloramientos, correspondientes a la parte alta del Miembro Inferior de la Formación Bajo Barreal, fue recuperada una vértebra caudal anficélica media a posterior (UNPSJB-Pv 595). La misma presenta una espina neural alta y comprimida lateralmente, $\mathrm{y}$ en vista lateral el centro vertebral presenta un contorno cuadrangular, rasgo anatómico considerado como autapomórfico para Andesaurus por Mannion \& Calvo (2011). Al respecto, Andesaurus es un saurópodo procedente del Cenomaniano-Turoniano (Leanza et al., 2004) de Neuquén y posicionado filogenéticamente en la base de Titanosauria (Curry Rogers, 2005; Mannion \& Calvo,
2011), por lo que este taxón es, en muchos casos, importante para interpretar la dinámica evolutiva de Titanosauria.

Dentro del registro fósil de dinosaurios saurópodos se destaca el hallazgo de un cráneo de Lithostrotia (MDT-Pv 2) con excepcional preservación en el yacimiento Cañadón Las Horquetas (Martínez, 1998a; Martínez et al., 2006, 2009) y recientemente nominado Sarmientosaurus musacchioi Martínez, Lamanna, Novas, Ridgely, Casal, Martínez, Vita \& Witmer, 2016. De este último sitio mencionado provienen además, la vértebra caudal anterior UNPSJB-Pv 580 inicialmente identificada como Diplodocidae indet. (Sciutto \& Martínez, 1994) y luego como Rebbachisauridae indet. por Ibiricu et al. (2012a) y recientemente como Limaysaurino (Ibiricu et al., 2015). Asimismo, Ibiricu et al. (2012a) asignan a Rebbachisauridae un conjunto de materiales caudales (UNPSJB-Pv 1004) que incluyen vértebras caudales anteriores y medias, y arcos hemales. Por último, también en el yacimiento Cañadón Las Horquetas, se hallaron numerosos restos del esqueleto axial (UNPSJBPv 1007) de un nuevo integrante de Rebbachisauridae: Katepensaurus goicoecheai (Ibiricu et al., 2013a; 2015). Este rebaquisáurido limaysaurino es hasta el momento el registro más austral a nivel genérico e incluye restos craneales, vértebras cervicales y caudales. Sin embargo, la morfología de las vértebras dorsales de Katepensaurus resulta especialmente novedosa particularmente por la presencia de rasgos anatómicos relacionados con la neumaticidad. Por su parte, los rebaquisáuridos del yacimiento de la estancia Ocho Hermanos son más escasos. Su registro, hasta el momento se acota a una vértebra cervical anterior-media (UNPSJB-Pv 1005; Ibiricu et al., 2012a).

Theropoda. El registro de dinosaurios terópodos en estos yacimientos de la Formación Bajo Barreal, está claramente dominado por los abelisáuridos (Tabla 1). Entre ellos se destacan los hallazgos de Xenotarsosaurus bonapartei estudiados por Martínez et al. (1986) con una particular fusión de los elementos tarsales, y de un maxilar izquierdo (Lamanna et al., 2002) que presenta una profusa decoración externa. En afloramientos de esta unidad en la provincia de Santa Cruz, fueron recuperadas una serie de seis vértebras asociadas (MPM99), incluyendo la cervical $10^{\circ}$, la dorsal $1^{\circ}$, un centro dorsal aislado y tres caudales de la porción media de la cola (Martínez et al., 2004b). Las vértebras son muy semejantes a las de otros abelisáuridos derivados (Carnotaurus sastrei, Aucasaurus garridoi) y son asignadas a Carnotaurini por Martínez et al. (2004b). Además, se han hallado numerosos dientes aislados de Abelisauroidea (Casal et al., 2009) y nuevos materiales en estudio pertenecientes posiblemente a un nuevo Abelisauridae (Martínez et al., 2005; Lamanna et al., 2012).

Se registran además, otros dinosaurios terópodos como el celurosaurio basal Aniksosaurus darwini por Martínez \& Novas (2006), restos de al menos dos megarraptóridos asignados a Megaraptor sp. (Lamanna et al., 2004), y la presencia de representantes de los clados Dromaeosauridae y Carcharodontosauridae documentada solamente al momento, por dientes aislados (Casal et al., 2009). No obstante, debe mencionarse que la mayoría de los dientes asignados a 
Dromaeosauridae en la Formación Bajo Barreal a partir de caracteres diagnósticos (Currie et al., 1990; Baszio, 1997; Sankey et al., 2002), fueron hallados en excavaciones donde se extrajeron restos de megarraptóridos desarticulados. Con esto, se interpreta que si bien los Dromaeosauridae pudieron haber participado en el sitio como carroñeros, no puede descartarse que esos dientes pertenezcan a los propios megarraptóridos. En este caso, estaríamos en un ejemplo de convergencia entre los dos taxones. Además, hasta el momento no se han hallado otros restos esqueletales que confirmen la pertenencia a Dromaeosauridae en estos niveles, mientras que los megarraptóridos son relativamente frecuentes.

Ornitischia. En estos niveles se destaca la presencia de Notohypsilophodon comodorensis conforme Martínez (1998b) e Ibiricu et al. (2014), el primer ornitópodo basal registrado en el Cretácico de Sudamérica. El hallazgo corresponde a un único individuo juvenil representado por buena parte del esqueleto, perteneciente a un grupo exitoso con una amplia distribución en tiempo y espacio, ya que han sido registrados desde el Jurásico Medio hasta el Cretácico Superior en Asia, Australia, Europa y Norteamérica.

Otros vertebrados no dinosaurios. El registro de vertebrados fósiles en los afloramientos cenomanianos-turonianos de la Formación Bajo Barreal incluye la presencia de las tortugas de agua dulce Chelidae Bonapartemys bajobarrealis y Prochelidella argentinae conforme Lapparent de Broin \& de la Fuente (2001). Esta última es considerada por los autores el quélido más antiguo registrado y presenta la retención de numerosos caracteres primitivos.

El registro fósil de la unidad se completa hasta el momento, con una fragmentaria falange del ala de Pterosauria indet. (Ibiricu et al., 2012b), restos no identificados de Crocodylia y placas de peces holósteos (Martínez et al., 2001) (Tabla 1).

\section{Síntesis estratigráfica de la Formación Lago Colhué Huapi}

La estratigrafía y aspectos cronológicos de los depósitos fluviales integrados por areniscas ocres y pelitas rojas, presentes en la margen Sur del codo del río Senguerr (Figuras 2, 4A), nacientes del río Chico (Figuras 2, 4B) y sectores de la costa del lago Colhué Huapi (Figura 3), en el Sur de la Provincia del Chubut, han sido históricamente confusos (Coria \& Cambiaso, 2007; Ibiricu et al., 2010; Casal et al., 2007, 2015b). De esta manera, en muchos casos resultó una conflictiva y dudosa ubicación estratigráfica de sus fósiles. En este sentido, Sciutto et al. (2008) refieren estos afloramientos al Miembro Superior de la Formación Bajo Barreal y la misma asignación a los dinosaurios procedentes de esos niveles (Casal et al., 2007; Ibiricu et al., 2010; Luna et al., 2003; Lamanna et al., 2003;

Tabla 1. Registro de dinosaurios y otros vertebrados de la Formación Bajo Barreal, Cuenca del Golfo San Jorge, Argentina.

Table 1. Record of dinosaurs and other vertebrates from the Bajo Barreal Formation, Gulf San Jorge Basin, Argentina.

\begin{tabular}{|c|c|c|}
\hline Taxon/materiales & Repositorio & Referencias \\
\hline Campylodon ameghinoi & MACN A-IOR63 & Huene (1929) \\
\hline Epachthosaurus sciuttoi & MACN-CH 1317 & Powell (1990) \\
\hline Epachthosaurus sciuttoi & UNPSJB-Pv 920 & Martínez et al. (2004a) \\
\hline Epachthosaurus sp. & MACN-CH 1317 & Casal \& Ibiricu (2010) \\
\hline Andesaurus sp. & UNPSJB Pv 525 & Powell et al. (1989) \\
\hline Sarmientosaurus musacchioi & MDT-Pv 2 & Martínez et al. (2016) \\
\hline Drusilasaura deseadensis & MPM-PV 2097 & Navarrete et al. (2011) \\
\hline Titanosaurios no epactosaurinos & UNPSJB-Pv 1010; 1010/1; 581 у 582 & Ibiricu et al. (2011) \\
\hline Rebbachisauridae indet. & UNPSJB-Pv 1004; 580; 1005 & Ibiricu et al. (2012a) \\
\hline Katepensaurus goicoecheai & UNPSJB-Pv 1007 & Ibiricu et al. (2013a) \\
\hline Megaraptor sp. & UNPSJB-Pv 958 y UNPSJB-Pv 944 & Lamanna et al. ( 2004) \\
\hline Aniksosaurus darwini & MDT-Pv 1 & Martínez \& Novas (2006) \\
\hline Xenotarsosaurus bonapartei & UNPSJB-Pv 194 & Martínez et al. (1986) \\
\hline Abelisauria indet., maxilar izquierdo & UNPSJB-Pv 247 & Lamanna et al. (2002) \\
\hline Abelisauroidea indet. & UNPSJB-Pv 1003 & Martínez et al. (2005) \\
\hline Abelisauridae indet. & MPM-99 & Martínez et al. (2004b) \\
\hline Abelisauroidea indet., dientes aislados & $\begin{array}{l}\text { UNPSJB-Pv } 221,224,225,226,229,230,231, \\
232,233,981,982,983,984,985,986 \text { y otros }\end{array}$ & Casal et al. (2009) \\
\hline Dromaeosauridae indet., dientes aislados & UNPSJB-Pv 988, 989 y 990 & Casal et al. (2009) \\
\hline Carcharodontosauridae indet., diente aislado & UNPSJB-Pv 969 & Casal et al. (2009) \\
\hline Notohipsylophodon comodorensis & UNPSJB-Pv 942 & Martínez (1998); Ibiricu et al. (2014) \\
\hline Chelonia indet., húmero & UNPSJB-Pv1008 & \\
\hline Prochelidella argentinae & MACN-CH-1680 & Lapparent de Broin \& de la Fuente (2001) \\
\hline Bonapartemys bajobarrealis & MACN-CH-1469 & Lapparent de Broin \& de la Fuente (2001) \\
\hline Chelonia indet., placas aisladas & UNPSJB-Pv 198, 200, 205 y otros & \\
\hline Crocodylia indet., placas aisladas & UNPSJB-Pv 199, 202, 203 y otros & \\
\hline Anura indet., fragmento de vértebra & UNPSJB-Pv 206 & \\
\hline Pterosauria indet. & UNPSJB-Pv 1009 & Ibiricu et al. (2012b) \\
\hline Holostei indet., placas aisladas & UNPSJB-Pv 207 y otros & \\
\hline
\end{tabular}


Prieto-Marquez \& Salinas, 2010). Otros autores asignaron a los dinosaurios procedentes de esa región a la Formación Laguna Palacios (Bonaparte, 1996; Novas, 1997) y hasta unidades inexistentes en la Cuenca del Golfo San Jorge como la Formación San Jorgé (sensu Brett Surman, 1979).

Por su parte, no menos confusas fueron las asignaciones estratigráficas de los dinosaurios procedentes de la margen Sur del codo del río Senguerr. Estos fueron asignados tanto al Miembro Superior de la Formación Bajo Barreal (Sciutto, 1981; Mannion \& Otero, 2012) como a la Formación Laguna Palacios (Bonaparte, 1978; Bonaparte \& Gasparini, 1979).

Recientemente, se caracterizaron de forma integrada la estratigrafía, la paleontología y la sedimentología de este intervalo estratigráfico del Cretácico Superior de la Cuenca del Golfo San Jorge lo que permitió la definición de una nueva unidad litoestratigráfica dentro del Grupo Chubut: la Formación Lago Colhué Huapi(Casal et al., 2015a)(Figura 1). Esta unidad se dispone por encima de la Formación Bajo Barreal y por debajo de la Formación Laguna Palacios, o bien de la Formación Salamanca (Maastrichtiano-Daniano sensu Barcat et al., 1989; Daniano temprano Clyde et al., 2014; Simeoni, 2014) de acuerdo a su posición en la cuenca. La base y el techo son transicionales, con excepción del contacto con la Formación Salamanca que en algunos casos es erosivo y en otros transicional (Casal et al., 2015a). En ocasiones, en la región del río Chico, la suprayace el basalto La Angostura que recientemente fue datado con ${ }^{40} \mathrm{Ar} / \mathrm{Ar}^{39}$ en $67,31 \pm 0,55 \mathrm{y}$ asignado al Maastrichtiano tardío (Clyde et al., 2014).

Al igual que otros depósitos del Grupo Chubut, las secuencias analizadas se interpretan como depósitos originados a partir de sistemas fluviales. En la localidad tipo de la Formación Lago Colhué Huapi se interpretó un paleoambiente fluvial con sistemas canalizados de alta sinuosidad y amplia planicie de inundación bien drenada, vinculados a un paleoclima semiárido (Allard \& Casal, 2013; Casal et al., 2015a). De acuerdo a los autores, se reconocen en la unidad cuerpos multiepisódicos que evidencian sistemas que reocupan su posición previa, erosionando parcial o totalmente los depósitos de abandono de canal. Asimismo, se describen casos donde la posición previa del canal no es reocupada. Estos canales abandonados serían el resultado del estrangulamiento de meandros y de procesos de avulsión regional y local (Miall, 1996; Bridge, 2003). Hacia el tope de la unidad, en algunos casos los canales abandonados son rellenados por el Basalto La Angostura (Maastrichtiano tardío).

\section{Edad de la Formación Lago Colhué Huapi}

Algunos integrantes de la fauna fósil exhumada en depósitos de la Formación Lago Colhué Huapi poseen un valor cronológico que permite asignarle a la unidad una edad Coniaciano-Maastrichtiano (Casal et al., 2015a). Esta edad es coherente con las interpretaciones surgidas a partir de las relaciones estratigráficas de la unidad, los datos radimétricos aportados por la subyacente Formación Bajo Barreal y el suprayacente Basalto La Angostura y datos bioestratigráficos de la Formación Salamanca.
Recientemente, Vallati et al. (en prensa) dieron a conocer una asociación palinológica procedente de pelitas laminadas $\mathrm{y}$ de color gris oscuro. Estas se hayan sobre una superficie erosiva en la parte superior de la Formación Lago Colhué Huapi y cubiertas por las típicas fangolitas rojas del tope de la unidad mencionada. Este registro incluye especies marcadoras del Maastrichtiano de la Provincia austral Proteacidites/ Nothofagidites como Quadraplanus brossus y Tubulifloridites lilliei, y también especies típicas del Maastrichtiano de la Provincia paleotropical Palmae como Buttinia andreevi y Gabonisporis vigourouxii conforme Vallati et al. (en prensa).

\section{Paleoclima de la Formación Lago Colhué Huapi}

En esta unidad el carácter paleohidrológico sugiere sistemas fluviales con importante variación estacional en la paleodescarga (Casal et al., 2015a). Además, en la sección media de la unidad se determinó mediante difracción de rayos X la presencia de arcillas como saponita y sepiolita en las planicies de inundación distales. Éstas se asocian con un clima semiárido con fluctuación del nivel freático (Zaaboub et al., 2005). Allí, se han reconocido también niveles de yeso (Casal, obs. pers.) y grietas de desecación (Casal et al., 2015a). Esto sugiere que luego del paleoclima cálido y húmedo de la Formación Bajo Barreal (Archangelsky et al., 1994; Pujana et al., 2007), se produjo una aridización del mismo durante la depositación de la sección inferior y media de la Formación Lago Colhué Huapi.

De acuerdo con Vallati et al. (en prensa), la asociación palinológica reconocida en la sección superior de la unidad incluye componentes característicos del Cretácico tardío de la Provincia paleotropical Palmae como Proxapertites y Spinizonocolpites. Esto sugiere un retorno a las condiciones más benignas hacia el Maastrichtiano tardío y que se extienden hasta el Daniano con veranos secos y primaveras lluviosas durante la sedimentación de la Formación Salamanca (Brea et al., 2005).

\section{Paleontología de la Formación Lago Colhué Huapi}

Los principales hallazgos de vertebrados fósiles de esta unidad proceden de la sección superior definida como el holoestratotipo de la unidad (Casal et al., 2015a) y los principales sitios de procedencia geográfica corresponden a una isla expuesta temporalmente en el sector Sudeste del lago Colhué Huapi y a las nacientes del río Chico (Figuras 2, 4B). Recientemente, se descubrió un nuevo afloramiento en Península Mocha (Estancia Kruger) (Figura 2), ubicada en la costa oriental del lago, caracterizado por la abundancia de restos, aunque hasta el momento, todos desarticulados. En estos sitios, y en el ya mencionado margen Sur del codo del río Senguerr (Figuras 2, 4A) se han recuperado restos de diversos taxones (Tabla 2) y que se sintetizan a continuación. Sauropoda. El primer hallazgo fue realizado a comienzos de la década de 1890, cuando Carlos Ameghino descubrió restos de un gran saurópodo titanosaurio a unos $5 \mathrm{~km}$ del margen izquierdo del río Chico, cerca de Pampa Pelada, en el Sur de Chubut (sensu Powell, 2003) (Figura 2). Ameghino asigna su procedencia a "areniscas rojas guaraníticas" y los materiales 

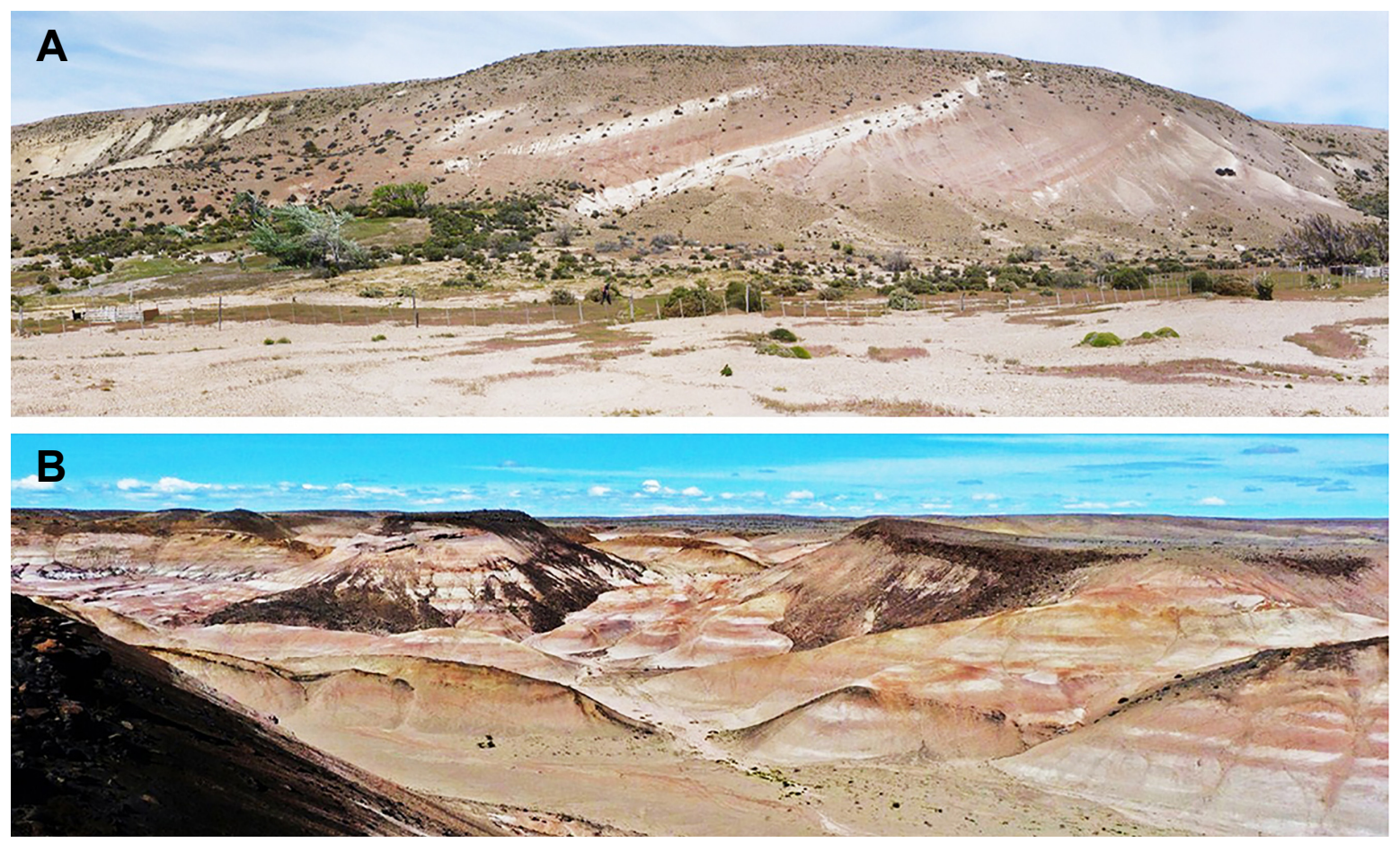

Figura 4. Afloramientos de la Formación Lago Colhué Huapi donde se observan las típicas fangolitas rojas: A, en el codo del río Senguerr; B, en las nacientes del río Chico, Sur de la Provincia del Chubut, Argentina.

Figure 4. Outcrops of the Lago Colhué Huapi Formation with the characteristic red fangolitas. A, in codo del río Senguerr; $\mathbf{B}$, in nacientes del río Chico South of Chubut Province, Argentina.

MLP 77-V-29-1 fueron interpretados como pertenecientes a Argyrosaurus superbus por Lydekker (1893), y posteriormente reestudiados por Huene (1929). Bonaparte \& Gasparini (1979) y Powell (2003) indican que su procedencia estratigráfica corresponde a la Formación Bajo Barreal, asignándole a la misma una edad senoniana.

Inicialmente, un posible nuevo ejemplar de Argyrosaurus superbus? (PVL 4628) fue recuperado de las areniscas amarillas del margen derecho del río Senguerr, en el borde Norte de la terraza fluvial Pampa María Santísima, en el límite provincial de Chubut y Santa Cruz (Powell, 2003) (Figuras 2, 4A). La procedencia estratigráfica de estos materiales fue referida a la parte inferior de la Formación Laguna Palacios (Bonaparte, 1978; Bonaparte \& Gasparini, 1979) y posteriormente Sciutto (1981) los ubica en la Formación Bajo Barreal. La edad de este sitio fue considerada la misma que para el holotipo de Argyrosaurus superbus (MLP 77V-29-1) hallado en los niveles del río Chico por Ameghino (Powell, 2003). Recientemente, a partir de un reestudio de estos materiales (PVL 4628), Mannion \& Otero (2012) los reasignaron a un nuevo género y especie dentro de Titanosauria nominado Elaltitan lilloi. Estudios de campo en la zona de procedencia de los materiales indican que Elaltitan lilloi proviene de la parte inferior de la Formación Lago Colhué Huapi, donde Casal et al. (2015a) definieron el paraestratotipo de esta nueva unidad litoestratigráfica. Consecuentemente, este saurópodo no sería contemporáneo con Argyrosaurus superbus.
En una isla parcialmente emergida en la parte Sudeste del lago Colhué Huapi (Figura 2), el Laboratorio de Paleovertebrados de la UNPSJB de Comodoro Rivadavia rescató varios restos fósiles (Tabla 2). Entre ellos, una serie caudal articulada de una nueva especie del titanosaurio derivado Aeolosaurus (Powell, 1987), nominado Aeolosaurus colhuehuapensis con relevancia en la información paleoambiental y cronológica (Casal et al., 2007). Posteriormente, Casal et al. (2010) dieron a conocer el hallazgo de un nuevo titanosaurio incompleto pero articulado (MDT-Pv 4) proveniente de la margen sur del río Chico (Figura 5), en niveles de areniscas fluviales de la Formación Lago Colhué Huapi. Entre los materiales recuperados se encuentran algunos osteodermos de forma piramidal y superficie altamente rugosa, pertenecientes a este ejemplar. Es el primer hallazgo de un dinosaurio con placas dérmicas hasta el momento en el Grupo Chubut (Tabla 2).

Theropoda. Al momento el registro de este clado en la Formación Lago Colhué Huapi es muy escaso (Tabla 2) y solo se han descubierto pocos restos fragmentarios, lo que marca un contraste con la abundancia de terópodos documentados en la Formación Bajo Barreal (Tabla 1). Se han recuperado hasta el momento un fragmento proximal de falange manual ungueal UNPSJB-Pv 1028 (Figura 6A) posiblemente de megarraptoriforme en una isla temporaria al Norte del lago (Figura 2), y recientemente otro elemento similar completo del digito I (Figura 6B) junto a un posible metatarso III también de este taxón en península Mocha. Estos 


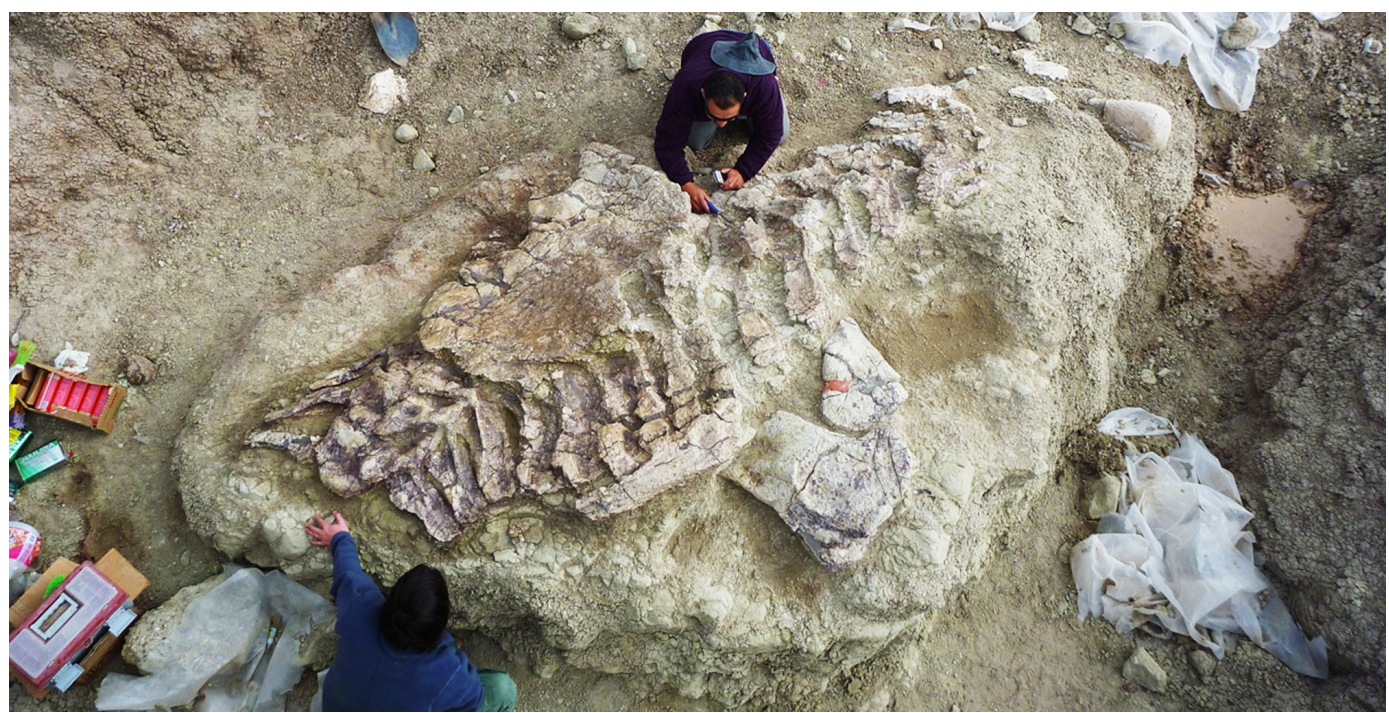

Figura 5. Vista en planta del Titanosauria parcialmente articulado procedente de la Formación Lago Colhué Huapi.

Figure 5. Plan view of a partially articulated Titanosauria from Lago Colhué Huapi Formation.

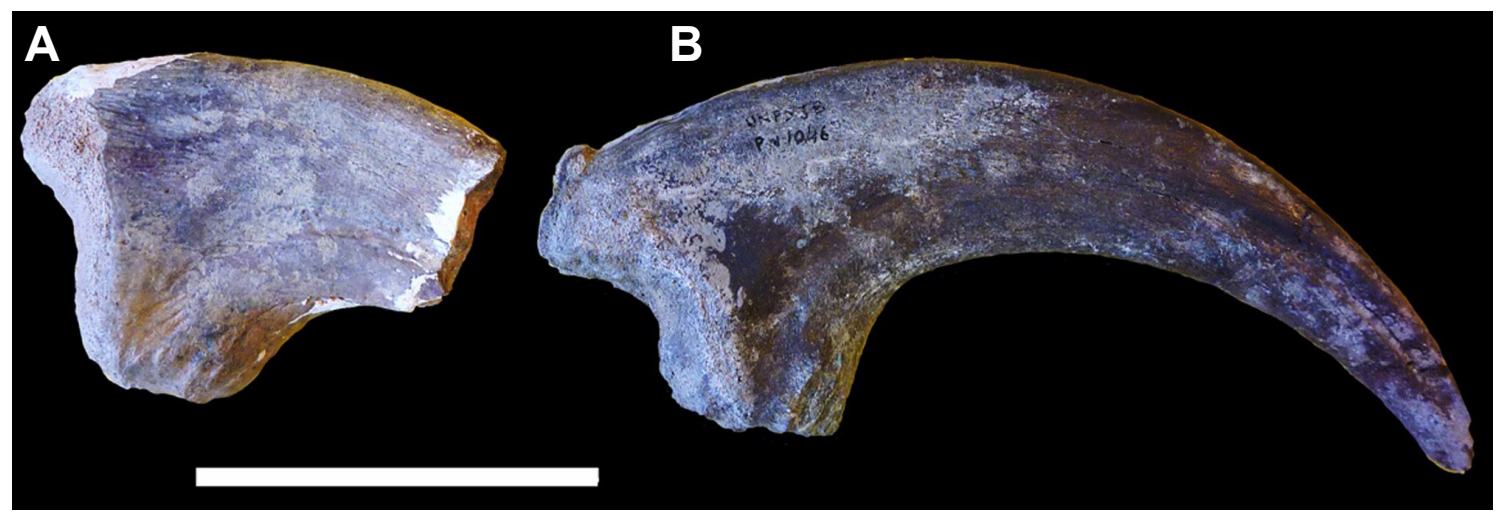

Figura 6. Falanges ungueales manuales de megarraptoriformes procedentes de la Formación Lago Colhué Huapi de la isla Norte del lago Colhué Huapi (A) y del Sudeste del mismo lago (B). Escala $=50 \mathrm{~mm}$.

Figure 6. Manus phalanges of megarraptoriformes from Lago Colhué Huapi Formation of the island North of the Colhué Huapi Lake (A) and of the Southeast of the Colhué Huapi Lake (B). Scale bar $=50 \mathrm{~mm}$.

hallazgos, junto a los realizados en la Formación Bajo Barreal y en otras unidades contemporáneas de Patagonia, muestra la abundancia de los megaraptóridos durante el intervalo Cenomaniano-Maastrichtiano. También se ha descubierto un diente con caracteres de dromaeosáurido (Currie et al., 1990; Baszio, 1997; Sankey et al., 2002; Casal et al., 2009).

Ornitischia. El registro de ornitópodos en esta unidad, es amplio y mucho más abundante que el de los niveles de la Formación Bajo Barreal, contrastando con lo mencionado para dinosaurios terópodos (Tabla 2). Tapia (1919) halló una rama mandibular izquierda de un ornitisquio, interpretado como un posible Ceratopsia, Notoceratops bonarelli, en el Noroeste del lago Colhué Huapi y que Bonaparte (1996) asignó al Maastrichtiano de la Formación Laguna Palacios. Posteriormente, Casal et al. (2006) sugirieron que su procedencia estratigráfica correspondería a los niveles de areniscas ocres y pelitas rojas del denominado por Sciutto et al. (2008) Miembro Superior de la Formación Bajo Barreal expuestos en los alrededores del lago Colhué Huapi. Actualmente, se asigna a la nueva Formación Lago Colhué Huapi. Notoceratops sería el único ceratopsio reconocido para Sudamérica hasta el momento. Sin embargo, diversos autores dudan de tal asignación (Dodson, 1990; Coria \& Cambiaso, 2007). Coria (com. pers.) considera la pertenencia de este material a una rama mandibular de Hadrosauridae. Desafortunadamente, el holotipo está extraviado, lo cual impide estudios complementarios del holotipo para confirmar o no su asignación a Ceratopsia.

En el año 1923 una comisión procedente del Field Museum of Natural History (Chicago, USA), recuperó restos de un dinosaurio en las nacientes del río Chico, aproximadamente a 4 km de la embocadura del río en el lago Colhué Huapi (Figura 2). Los mismos pertenecen a un hadrosáurido basal que Brett 
Surman (1979) nominó como Secernosaurus koerneri. PrietoMarquez \& Salinas (2010) confirman la asignación a este clado y la misma interpretación fue realizada por Coria \& Cambiaso (2007). Estos últimos autores, además, sugieren la presencia de más de un individuo dentro de los materiales colectados por la expedición americana de 1923. Lamentablemente, estos históricos descubrimientos no fueron acompañados de estudios estratigráficos precisos. De hecho, el esqueleto de Secernosaurus koerneri, fue asignado como proveniente de la Formación San Jorgé (sic), la cual no existe para el Cretácico de la Cuenca del Golfo San Jorge. Posiblemente tal asignación se fundamentó en la denominación dada por Windhausen (1918) a la actual Formación Salamanca como Formación San Jorge (Uliana \& Legarreta, 1999, p. 508), la cual también está expuesta en el sitio de hallazgo de Secernosaurus. Asimismo, Bonaparte (1996) y Novas (1997) lo asignan a la Formación Laguna Palacios, Weishampel \& Horner (1990) a la Formación Bajo Barreal. Prieto-Marquez \& Salinas (2010) se basan en la asignación dada por Casal et al. $(2006,2007)$ al Miembro Superior de la Formación Bajo Barreal de Sciutto et al. (2008). Por estas continuas controversias sobre su procedencia estratigráfica, diversos investigadores excluyeron a Secernosaurus de sus estudios bioestratigráficos (González Riga \& Casadío, 2000). No obstante, en base a los estudios de campo en la región de procedencia geográfica en el río Chico en el Sur de Chubut y al posible hallazgo recientemente de su antigua excavación, actualmente se asigna a la nueva Formación Lago Colhué Huapi.

Como fue mencionado anteriormente, en los últimos años se han incrementado los estudios geológicos y paleontológicos de estas secuencias sedimentarias. Esto condujo a nuevos e importantes hallazgos de fósiles realizados durante las dos últimas décadas (Tabla 2). Al respecto, Luna et al. (2003) dieron a conocer un ilion de ornitópodo (UNPSJB-Pv 973) procedente de la isla del sudeste del lago Colhué Huapi
(Figura 2), y que podría corresponder a un hadrosáurido. Del mismo sitio proceden restos de un ornitópodo iguanodóntido [UNPSJB-Pv 960 (Ibiricu et al., 2010)]. Recientemente, en el sitio denominado península Mocha (Figura 2) se recuperaron materiales de Ornithopoda indet. que incluyen vértebras caudales incompletas y un extremo distal de fémur.

En las nacientes del río Chico se recuperaron una serie de restos (UNPSJB Pv 1050) que incluyen vértebras dorsales, sacras y caudales incompletas, junto a fragmentos de surcos alveolares con características de un hadrosáurido. Estos materiales provienen de un intervalo estratigráfico ubicado por encima de una incisión erosiva labrada sobre la Formación Lago Colhué Huapi y están incluidos en areniscas y pelitas que no tienen las características diagnósticas de la unidad mencionada. Esto sugiere preliminarmente que el hadrosáurido correspondería a otra unidad fuera del Grupo Chubut. Actualmente se están realizando estudios estratigráficos y de nuevos restos hallados de palinomorfos y estromatolitos que contribuirán al entendimiento de la procedencia del hadrosáurido.

Otros vertebrados no dinosaurios. En una isla del Norte del lago Colhué Huapi (Figura 3) se halló una escápula fragmentaria de quelonio (UNPSJB-Pv 1029). En otra isla al Sudeste del mismo lago, se recuperaron dos ramas mandibulares incompletas (UNPSJB-Pv 961) de crocodyliforme posiblemente un Mesoeucrocodylia (Lamanna et al., 2003). Recientemente se descubrió el primer registro de pez dipnoo a través de una placa dentaria perfectamente preservada UNPSJB-Pv 1047 (Figura 7).

\section{DISCUSIÓN}

Las características litológicas, estratigráficas y paleontológicas del registro más moderno del Grupo Chubut en la Cuenca del Golfo San Jorge, permitieron escindir una nueva

Tabla 2. Registro de dinosaurios y otros vertebrados de la Formación Lago Colhué Huapi, Cuenca del Golfo San Jorge, Argentina.

Table 2. Record of dinosaurs and other vertebrates from the Lago Colhué Huapi Formation, Gulf San Jorge Basin, Argentina.

\begin{tabular}{lll}
\hline Taxón/material & Repositorio & Referencia \\
\hline Notoceratops bonarelli & DNM no catalogado & Tapia (1918) \\
Secernosaurus koerneri & FMNH PP13423 & Brett-Surman (1979) \\
Hadrosauridae indet. & UNPSJB-Pv 1050 & \\
Ornithopoda indet. & UNPSJB-Pv 973 & Luna et al. $(2003)$ \\
Ornithopoda indet. & UNPSJB-Pv 960 & Ibiricu et al. $(2010)$ \\
Ornithopoda indet., vértebras & UNPSJB-Pv 1034,1043 & \\
Argyrosaurus superbus & MLP 77-V-29-1 & Lydekker (1893) \\
Aeolosaurus colhuehuapensis & UNPSJB-Pv 956 & Casal et al. $(2007)$ \\
Titanosauria indet. & MDT-Pv 4 & Casal et al. $(2010)$ \\
Titanosauria indet. & UNPSJB-Pv 1051 & \\
Titanosauria indet., vértebra & UNPSJB-Pv 1033 & \\
Elaltitan lilloi & PVL-4628 & Mannion \& Otero (2012) \\
Theropoda indet., fragmento falange ungueal & UNPSJB-Pv 1028 & \\
Theropoda indet., falange ungueal & UNPSJB-Pv 1046 & \\
Ave indet., restos fragmentarios & UNPSJB-Pv 1049 & \\
Dipnoi indet., placa dentaria & UNPSJB-Pv 1047 & \\
Chelonia indet. & UNPSJB-Pv 1029 & \\
\hline
\end{tabular}




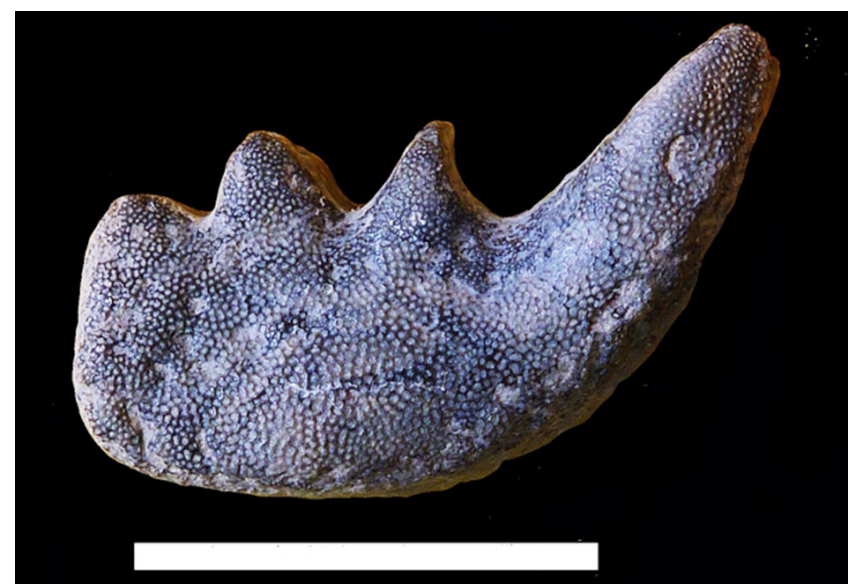

Figura 7. Placa dentaria de Dipnoi indet. procedente de la Formación Lago Colhué Huapi. Escala $=10 \mathrm{~mm}$.

Figure 7. Dental plate of Dipnoi indet. from Lago Colhué Huapi Formation. Scale bar $=10 \mathrm{~mm}$.

unidad litoestratigráfica dentro del grupo, la Formación Lago Colhué Huapi (Casal et al., 2015a). Esto permite ordenar estratigráfica y cronológicamente el contenido paleontológico de estos niveles, contribuyendo al entendimiento de la evolución de la fauna cretácica de la cuenca y sus vinculaciones con otras cuencas patagónicas y de Gondwana en general. En este sentido, el registro fósil recuperado hasta el momento en la Formación Bajo Barreal (Cenomaniano temprano-Turoniano tardío) en Sierra San Bernardo y Sierra Nevada, como principales sitos de procedencia de vertebrados fósiles, claramente se diferencia de la registrada en afloramientos de la suprayacente Formación Lago Colhué Huapi (Coniaciano-Maastrichtiano), presentando la primera de ellas formas más basales para sus respectivos grupos.

Recientes estudios faunísticos basados en análisis estadísticos de similitud (Canale et al., 2011), vinculan en este aspecto al Miembro Inferior de la Formación Bajo Barreal con el Subgrupo Río Limay (formaciones Candeleros y Huincul), depositado durante el Cretácico Superior temprano (Cenomaniano-Turoniano), mientras el mismo patrón estadístico resultó para los afloramientos de la Formación Lago Colhué Huapi y con respecto a la Formación Allen, depositada durante el Cretácico Superior tardío (CampanianoMaastrichtiano).

Entre los taxones reconocidos en la Formación Bajo Barreal están los rebaquisáuridos, que constituyen el último clado superviviente de Diplodocoidea (Apesteguía, 2002; Whitlock, 2011). Su presencia en la Formación Bajo Barreal reviste importancia tanto a nivel cronológico como paleobiogeográfico. En Sudamérica, su registro está acotado al período de tiempo comprendido entre el Barremiano y el Turoniano, donde parece haber tenido lugar la extinción de los Rebbachisauridae en particular y diplodocoideos en general (Salgado et al., 2004; Coria \& Salgado, 2005; Apesteguía, 2007; Ibiricu et al., 2012a, 2013a). Los rebaquisáuridos co-existieron con los titanosaurios basales durante el Cretácico temprano y medio, aunque posiblemente ocupando diferentes nichos ecológicos. Sin embargo, solamente las formas más derivadas de este último grupo alcanzaron el Cretácico Superior tardío.

El mismo patrón o "turnover" mencionado para los rebaquisáuridos y titanosaurios fue propuesto para los terópodos carcarodontosáuridos y los abelisáuridos (Apesteguía, 2002; Leanza et al., 2004; Coria \& Salgado, 2005). Mientras que durante el Cretácico temprano ambos taxones fueron contemporáneos (Novas, 2009), solamente el último de ellos continuó hasta fines del Cretácico, mientras los carcarodontosáuridos se extinguían hacia mediados del Cretácico tardío (Turoniano: Apesteguía, 2002; Novas et al., 2005; Coria, 2007).

Los abelisáuridos son uno de los grupos más abundantes de terópodos en Argentina, lo cual resalta su importancia paleogeográfica y cronológica (Novas, 2009). Esta diversidad taxonómica se ve también particularmente reflejada en Gondwana. El registro de terópodos en estos yacimientos cenomanianos-turonianos de la Formación Bajo Barreal está dominado por terópodos abelisáuridos. No obstante, para niveles contemporáneos de la Cuenca Neuquina son dominantes los carcarodontosáuridos, mientras que los abelisaurios lo hacen a partir del Santoniano (Apesteguía, 2002; Leanza et al., 2004).

El maxilar izquierdo UNPSJB-Pv 247 asignado a Abelisauria por Lamanna et al. (2002) presenta una profusa decoración externa que lo relacionan estrechamente con el abelisáurio basal Rugops primus de Sereno et al. (2004), proveniente de la Formación Echkar del Cenomaniano de Nigeria, África. Asimismo, Martínez et al. (1993) y posteriormente Lamanna et al. (2002) mencionan que dicho maxilar muestra estrechas relaciones con otros abelisaurios como Majungasaurus crenatissimus (Depéret, 1896; Lavocat, 1954; ver Krause et al., 2007) e Indosuchus raptorius (Chatterjee, 1978), ambos del Cretácico Superior de Madagascar e India respectivamente. Esto, se vincula a una posible ruta de dispersión como la propuesta para el Cretácico Superior por Sampson et al. (1998) entre IndiaMadagascar, Antártida-Australia y Sudamérica. De hecho, el hallazgo de abelisaurios tempranos en Chubut permite pensar en una antigua distribución "pan-gondwánica" de los Abelisauridae (con conexión terrestre entre Sudamérica y África hasta finales del Cretácico temprano), la que también estaría en consonancia con el registro en la Formación Bajo Barreal (Lamanna et al., 2002).

Otro de los grupos de terópodos con representación taxonómica en las cuencas patagónicas son los coelurosaurios. En la Formación Bajo Barreal se destaca Aniksosaurus darwini, un celurosaurio basal no relacionado cercanamente con otros terópodos hallados en la misma unidad ni con otras formas documentadas de Argentina. Este pequeño terópodo está representado por cinco ejemplares y posiblemente tenía un comportamiento gregario (Martínez \& Novas, 2006; Ibiricu et al., 2013b). Además, representaría durante el Cretácico Superior un superviviente de una radiación celurosauriana basal pobremente documentada hasta el momento (Martínez \& Novas, 2006). Las comparaciones efectuadas por estos 
autores están coherentemente enfocadas en formas no pertenecientes al Cretácico Superior, como Sinosauropteryx del Jurásico Superior o Cretácico Inferior de China (Currie \& Chen, 2001); Nqwebasaurus thwasi del BerriasianoValanginiano de Sudáfrica (de Klerk et al., 2000); Sinraptor heping del Jurásico Superior de China (Currie \& Zhao, 1993) y Allosaurus, Ornitholestes hermanni y Tanycolagreus topwilsoni estos tres últimos del Jurásico Superior de Estados Unidos (Osborn, 1903; Gilmore, 1920; Carpenter et al., 2005). Novas et al. (2012), dieron a conocer un nuevo celurosaurio basal de la Formación Candeleros, Bicentenaria argentina. Este pequeño dinosaurio terópodo representaría otro de los linajes basales de celurosaurios que habitaron Patagonia durante el Cenomaniano. Asimismo, basados en distintas evidencias, Bicentenaria, al igual que Aniksosaurus, podría haber tenido un comportamiento gregario (Novas et al., 2012; Ibiricu et al., 2013b). Esto apoyaría que este tipo de comportamiento está presente tempranamente en la evolución de Coelurosauria.

La fauna de ornitisquios en la Formación Bajo Barreal está representada únicamente por Notohypsilophodon comodorensis. Este pequeño ornitópodo basal no driomorfo es el representante más antiguo del clado en afloramientos cretácicos sudamericanos (Martínez, 1998b; Novas, 2009; Ibiricu et al., 2014). Los ornitópodos basales presentan una distribución geográfica global, sin embargo la anatomía postcraneal de $N$. comodorensis muestra similitudes con materiales descriptos tanto en Australia como en Antártida (Martínez, 1998b; Agnolin et al., 2010). Estas similitudes sugieren cierta relación de la Cuenca del Golfo San Jorge con Australia vía Antártida durante el Cretácico Superior temprano (Martínez, 2007). Sin embargo, en parte debido a la naturaleza fragmentaria de muchos de los representantes del grupo y también a la falta de resolución filogenética a nivel intra-especifico, las relaciones paleobiogeográficas de ornitópodos basales permanece aún no definidas (Norman et al., 2004; Benson et al., 2012). No obstante, la posibilidad de "un corredor" relacionando Australia, Antártida y Sudamérica es una interpretación posible (Martínez et al., 2008; Poropat et al., 2015), ya demostrada para otros taxones.

Por su parte, el registro fósil de la Formación Lago Colhué Huapi (Coniaciano-Maastrichtiano), que procede principalmente de la región conformada por el lago Colhué Huapi-nacientes del río Chico y en menor medida del margen Sur del codo del río Senguerr, contiene taxones considerados como más derivados evolutivamente que los procedentes de niveles de la Formación Bajo Barreal (Casal et al., 2007).

En cuanto a la fauna de dinosaurios saurópodos se destaca la presencia del género Aeolosaurus, cuyo registro se restringe actualmente al Campaniano-Maastrichtiano de las formaciones Angostura Colorada (Powell, 1987), Allen (Salgado \& Coria, 1993), Los Alamitos (Salgado et al., 1997b) y Loncoche (González Riga, 1995,1999; Previtera \& González Riga, 2008), todas ellas parcial o completamente coetáneas. El registro sudamericano de dicho taxón se extiende a Brasil, ya que existen registros en la Formación Adamantina (Campaniano-Maastrichtiano, sensu Gobbo
Rodrigues et al., 1999) en el Estado de São Paulo (Bertini et al., 1999a, Santucci \& Arruda Campos, 2011) y en la base del Miembro Serra da Galga de la Formación Marília (Maastrichtiano inferior) en el Estado de Minas Gerais (Bertini et al., 1999b). Sin embargo, las relaciones de los Aeolosaurinae del Cretácico Superior de Brasil y Argentina y en particular las relaciones entre las distintas especies de Aeolosaurus, ha sido recientemente discutida por Martinelli et al. (2011). Por otra parte, las unidades arriba mencionadas de la Cuenca Bauru en Brasil, han sido cronológicamente correlacionadas con aquellas del Cretácico Superior de la Patagonia Argentina (Huene, 1929, 1932; Candeiro et al., 2006a,b; Candeiro, 2010), incluyendo erróneamente en algunos casos, los niveles de la Formación Bajo Barreal.

El registro fósil de hadrosáuridos en Sudamérica es escaso $\mathrm{y}$ en muchos casos fragmentario, en comparación con aquel recuperado en Asia y Norteamérica (Prieto-Marquez \& Salinas, 2010). Sin embargo, su presencia es relevante por sus implicancias paleobiogeográficas y evolutivas (Casamiquela, 1964; Bonaparte et al., 1984; Coria, 2010; Prieto-Márquez \& Salinas, 2010; Coria et al., 2012).

Por su parte, los hadrosáuridos registrados en Patagonia se encuentran restringidos a sedimentos campanianosmaastrichtianos de la Formación Allen (González Riga \& Casadío, 2000; Juarez Valieri et al., 2010; Coria et al., 2012), la Formación Los Alamitos (Bonaparte et al., 1984) y la Formación Paso del Sapo (Apesteguía \& Cambiaso, 1999; Apesteguía et al., 2012). Además, en este trabajo, se define la procedencia estratigráfica de Secernosaurus koerneri como de la parte superior de la Formación Lago Colhué Huapi (Brett Surman, 1979), a partir de los datos geográficos con que se cuenta y al posible hallazgo de su excavación que está en proceso de reapertura actualmente. La presencia de este taxón en Patagonia es atribuido a fenómenos de geodispersión desde Norteamérica hacia finales del Cretácico (Coria, 2010, 2012; Prieto-Marquez, 2010a; Coria et al., 2012). En este sentido, Apesteguía et al. (2012) consideran que los hadrosáuridos están ausentes en unidades del Campaniano inferior y medio, y son abundantes en el Campaniano superior. Asimismo, estos autores también explican la ausencia del taxón en las unidades del Cretácico tardío de la Cuenca Bauru, sugiriendo una dispersión occidental por Sudamérica, lo que argumentan por datos icnológicos.

En Patagonia, los hadrosáuridos junto a Aeolosaurus son interpretados como miembros de una asociación faunística para el Cretácico Superior (Campaniano-Maastrichtiano) (Bonaparte, 1984; 1986). Son ejemplos de esto las asociaciones dadas por Kritosaurus australis de Bonaparte et al. (1984), posiblemente una sinonimia de Secernosaurus conforme Prieto-Marquez \& Salinas (2010), y Aeolosaurus sp. en niveles de la Formación Los Alamitos (Salgado et al., 1997b), y Willinakaqe salitralensis de Juárez Valieri et al. (2010) con Aeolosaurus sp. conforme Salgado \& Coria (1993) en niveles de la Formación Allen (Provincia de Río Negro). Esta asociación, considerada como válida para determinar la edad-Vertebrados Alamitense asignada al Campaniano (Bonaparte, 1992), fue confirmada por Salgado et al. (1997b). La presencia de estos taxones permitió corroborar 
la correlación dada por Bonaparte (1992) entre las formaciones Los Alamitos, Allen y Angostura Colorada, y también con la Formación Loncoche (Candeiro, 2010) en la Cuenca Neuquina. Consecuentemente, la asociación registrada en el Sur de Chubut entre Aeolosaurus colhuehuapensis y Secernosaurus koerneri permite correlacionar las secuencias fluviales de la parte superior de la Formación Lago Colhué Huapi con las unidades de la Cuenca Neuquina mencionadas anteriormente. La edad de esos niveles superiores de la Formación Lago Colhué Huapi correspondientes al Campaniano-Maastrichtiano sugiere preliminarmente que es posible extender esta correlación, al menos de forma parcial, a las formaciones La Colonia y Paso del Sapo del Cretácico tardío de la Cuenca Cañadón Asfalto.

Finalmente, se considera que los hadrosaurios Secernosaurus koerneri y UNPSJB-Pv 1050, y el dudoso ceratopsio (Tapia, 1919) estarían vinculados al intercambio faunístico ocurrido al menos desde el Campaniano temprano a través de puentes geográficos entre América del Norte y América del Sur (Bonaparte et al., 1984; Bonaparte, 1986, Salgado \& Coria, 1996; Coria, 1999; Prieto-Marquez \& Salinas, 2010; Coria et al., 2012), junto a lagartos Teiidae y mamíferos Eutheria (Bonaparte, 1986).

\section{CONCLUSIONES}

En este trabajo se ordena y posiciona estratigráfica y cronológicamente la fauna de vertebrados recuperados hasta el momento en las secuencias sedimentarias del Cretácico Superior (Cenomaniano-Maastrichtiano) del Grupo Chubut, expuestas en el centro-Sur de la Provincia del Chubut, Patagonia Argentina. Esto, permite escindir el registro fósil procedente de la Formación Bajo Barreal (Cenomaniano tempranoTuroniano tardío) y de la Formación Lago Colhué Huapi (Coniaciano-Maastrichtiano). De esta manera se esclarecen interpretaciones históricamente confusas y erróneas para este intervalo estratigráfico de la Cuenca del Golfo San Jorge.

Los elementos faunísticos de la Formación Bajo Barreal presentan relaciones uniformes y coherentes con formas de Gondwana para del Cretácico Superior bajo y fundamentalmente, con la fauna procedente de las formaciones Candeleros y Huincul de la Cuenca Neuquina. Mientras que el registro fósil de la parte superior de la Formación Lago Colhué Huapi expuesta en la costa oriental del lago homónimo y en nacientes del río Chico, es más afín con las formaciones Allen, Los Alamitos, Loncoche y Angostura Colorada de la Cuenca Neuquina, y las formaciones Paso del Sapo y La Colonia de la Cuenca Cañadón Asfalto.

En su conjunto, los resultados de este trabajo son de importancia para caracterizar las relaciones de la fauna en estos niveles no solo para el resto de las cuencas patagónicas, sino también para niveles contemporáneos de Gondwana.

\section{AGRADECIMIENTOS}

Los autores agradecen a E. Ivany, E. González y E. Balverdi por su trabajo de campo en diversas campañas y en laboratorio. A los propietarios de las estancias visitadas $\mathrm{H}$.
Martínez, "Chiche" Martínez, A. Perujo (†), L. Insúa, L. y R. Kruger, familia Goicoechea y E. Valbuena. A H. Hourcade y la comunidad de Buen Pasto. A J. Crivelli de la Municipalidad de Sarmiento por toda su colaboración en la logística de campo. A J. Allard y N. Foix por su constante aporte sobre la geología de la región. Uno de los autores (G.A.C.) agradece al Departamento de Geología de la UNPSJB por facilitar el vehiculo para las campañas realizadas durante la tesis doctoral. A G.S. Libardi por la traducción del resumen al portugués. A A. de Sosa Tomas por su colaboración en las figuras. A los revisores del trabajo L. Salgado y S. Apesteguía que con sus críticas permitieron mejorar esta contribución. A la Agencia Nacional de Promoción Científica y Técnica (PICT 2012-2884 a L.M. Ibiricu) por el financiamiento para distintas campañas en el centro sur de la Provincia de Chubut).

\section{REFERENCIAS}

Allard, J.O. \& Casal, G.A. 2013. Análogos de afloramientos del Cretácico Superior en las nacientes del río Chico: sedimentología, paleoambiente, características geométricas y heterogeneidades intra-reservorio. In: JORNADAS GEOLÓGICAS DE LA CUENCA DEL GOLFO SAN JORGE, 1, 2013. Actas, Comodoro Rivadavia, p. 17-18.

Agnolin, F.L.; Ezcurra, M.D.; Pais, D.F. \& Salisbury, S.W. 2010. A reappraisal of the Cretaceous non-avian dinosaur faunas from Australia and New Zealand: evidence for their Gondwanan affinities. Journal of Systematic Palaeontology, 8:257-300. doi:10.1080/14772011003594870

Apesteguía, S. 2002. Gran Gondwana y la fauna de vertebrados del Mar de Kawas (Campaniano-Maastrichtiano). In: SIMPÓSIO SOBRE EL CRETÁCICO DE AMÉRICA DEL SUR, 2, 2002. Actas, São Pedro, p. 143-147.

Apesteguía, S. 2007. The sauropod diversity of the La Amarga Formation (Barremian), Neuquén (Argentina). Gondwana Research, 12:543-546. doi:10.1016/j.gr.2007.04.007

Apesteguía, S. \& Cambiaso, A.V. 1999. Registro de hadrosaurios en la Formación Paso del Sapo, del Campaniano-Maastrichtiano de Chubut, su importancia bioestratigráfica y sus posibilidades de correlación con unidades contemporáneas. Ameghiniana, 36: 26R.

Apesteguía, S.; Cambiaso, A.V. \& Agnolin, F. 2012. Vertebrados de la Formación Paso del Sapo (Campaniano/Maastrichtiano), Provincia de Chubut, Argentina. Ameghiniana, 49:395-400. doi:10.5710/AMGH.v49i3(358)

Archangelsky, S.; Bellosi, E.; Jalfin, G. \& Perrot, C. 1994. Palynology and Alluvial facies from the Mid-Cretaceous of Patagonia, Subsurface of San Jorge Basin, Argentina. Cretaceous Research, 15:127-142. doi:10.1006/cres.1994.1006

Barcat, C.; Cortiñas, J.S.; Nevistic, V.A. \& Zucchi, H.E. 1989. Cuenca Golfo San Jorge. In: G.A. Chebli \& L.A. Spalletti (eds.) Cuencas Sedimentarias Argentinas. Tucumán, Universidad Nacional de Tucumán, p. 319-345 (Correlación Geológica 6).

Barreda, V. \& Archangelsky, S. 2006. The southernmost record of tropical pollen grains in the mid-Cretaceous of Patagonia, Argentina. Cretaceous Research, 27:778-787. doi:10.1016/j. cretres.2006.02.002

Baszio, S. 1997. Systematic palaeontology of isolated dinosaur teeth from the latest Cretaceous of south Alberta, Canada. Courier Forschungsinstitut Senckenberg, 196:33-77.

Bellosi, E.S.; Palamarczuk, S.; Barreda, B.; Sanagua, J. \& Jalfin, G. 2000. Litofacies y palinología del contacto Grupo Chubut- 
Formación Salamanca en el oeste de la cuenca del Golfo San Jorge. In: SIMPOSIO ARGENTINO DE PALEOBOTÁNICA Y PALINOLOGÍA, 11, 2000. Actas, San Miguel de Tucumán, APA, p. 9.

Benson, R.B.J.; Rich, T.H.; Vickers-Rich, P. \& Hall, M. 2012. Theropod fauna from southern Australia indicates high polar diversity and climate-driven dinosaur provinciality. PLOS ONE, 7:e37122. doi:10.1371/journal.pone.0037122

Bertini, R.J.; Santucci, R.M. \& Arruda-Campos, A.C. 1999a. First occurence of Aeolosaurus (Sauropoda, Titanosauridae) in Bauru Group of the Paraná Basin. Brazil. In: CONGRESSO BRASILEIRO DE PALEONTOLOGÍA, 16, 1999. Boletim de Resumos, Crato, URCA, p. 27-28.

Bertini, R.J.; Santucci, R.M. \& Ribeiro, L.C.B. 1999b. O titanossáurido Aeolosaurus sp. (Saurischia, Sauropoda) no Membro Serra da Galga da Formação Marília. Grupo Bauru do Triângulo Mineiro. In: SIMPÓSIO DE GEOLOGÍA DO SUDESTE, 6, 1999. Boletim de Resumos, São Pedro, UNESP, p. 78.

Bonaparte, J.F. 1978. El mesozoico de América del Sur y sus tetrápodos (geología, reptiles). Opera Lilloana, 26:1-596.

Bonaparte, J.F. 1984. El intercambio faunístico de vertebrados continentales entre America del Sur y del Norte a fines del Cretácico. In: CONGRESOLATINOAMERICANODEPALEONTOLOGÍA, 3, 1984. Memorias, Ciudad de México, p. 38-45.

Bonaparte, J.F. 1986. History of the terrestrial Cretaceous vertebrates of Gondwana. In: CONGRESO ARGENTINO DE PALEONTOLOGÍA Y BIOESTRATIGRAFÍA, 4, 1986. Actas, Mendoza, p. 63-95.

Bonaparte, J.F. 1992. Una nueva especie de Triconodonta (Mammalia), de la Formación Los Alamitos, provincia de Río Negro y comentarios sobre su fauna de vertebrados. Ameghiniana, 29:99-110.

Bonaparte, J.F. 1996. Dinosaurios de América del Sur. Buenos Aires, Artes Gráficas Sagitario, $174 \mathrm{p}$.

Bonaparte, J.F.; Franchi, M.; Powell, J.E. \& Sepúlveda, E. 1984. La Formación Los Alamitos (Campaniano-Maastrichtiano) del sudeste de Río Negro. Con descripción de Kritosaurus Australis n. sp. (Hadrosauridae). Significado paleogeográfico de los vertebrados. Revista de la Asociación Geológica Argentina, 49:284-299.

Bonaparte, J.F. \& Gasparini, Z.B. 1979. Los saurópodos de los Grupos Neuquén y Chubut, y sus relaciones cronológicas. In: GONGRESO GEOLÓGICO ARGENTINO DE PALEONTOLOGÍA Y BIOESTRATIGRAFÍA, 7, 1979. Actas, Neuquén, p. 393-406.

Brea, M.; Matheos, S.; Zamuner, A. \& Ganuza, D. 2005. Análisis de los anillos de crecimiento del bosque fósil de Víctor Szlápelis, Terciario inferior del Chubut, Argentina. Ameghiniana, 42:407-418.

Brett Surman, M.K. 1979. Phylogeny and palaeobiogeography of hadrosaurian dinosaurs. Nature, 277:560-562. doi:10.1038/ $277560 a 0$

Bridge, J.S. 2003. Rivers and floodplains: forms, processes and sedimentary record. Oxford, Blackwell, $491 \mathrm{p}$.

Bridge, J.; Jalfin, G. \& Georgieff, S. 2000. Geometry, lithofacies, and distribution of Cretaceous fluvial sandstone bodies, San Jorge Basin, Argentina: Outcrop analogue for the hydrocarbon-bearing Chubut Group. Journal Sedimentary Research, 70:341-359. doi:10.1306/2DC40915-0E47-11D7-8643000102C1865D

Canale, J.; Ibiricu, L.M.; Haluza, A. \& Casal, G. 2011. The continental Cenomanian of Patagonia: a comparison between the fossil vertebrate faunas of the río Limay Subgroup (Neuquén Group) and the Bajo Barreal Formation (Chubut Group) fossil vertebrate faunas. Ameghiniana, 48:98R.
Candeiro, C.R.A. 2010. Record of the genus Aeolosaurus (Sauropoda, Titanosauria) in the Late Cretaceous of South America: paleogeographic implications. Estudios Geológicos, 66:243-253. doi: 10.3989/egeol.40338.081

Candeiro, C.R.A.; Martinelli, A.G.; Avilla, L.S. \& Rich, T. 2006 a. Tetrapods from the Upper Cretaceous (Turonian Maastrichtian) Bauru Group of Brazil: a reappraisal. Cretaceous Research, 27:923-946. doi:10.1016/j.cretres.2006.05.002

Candeiro, C.R.A.; Santos, A.R.; Rich, T.H.; Marinho, T.S. \& Oliveira, E.C. 2006b. Vertebrate fossils from the Adamantina Formation (Late Cretaceous), Prata paleontological District, Minas Gerais State, Brazil. Geobios, 39:319-327. doi:10.1016/j. geobios.2005.10.003

Casal, G.A.; Allard, J.O. \& Foix, N. 2015. Análisis estratigráfico y paleontológico de afloramientos del Cretácico Superior en la cuenca del Golfo San Jorge: propuesta de nueva unidad litoestratigráfica para el Grupo Chubut. Revista de la Asociación Geológica Argentina, 72:81-99.

Casal, G.A.; Candeiro, C.R.; Martinez, R.D.; Ivany, E. \& Ibiricu, L.M. 2009. Dientes de Theropoda (Dinosauria: Saurischia) de la Formación Bajo Barreal, Cretácico Superior, Provincia del Chubut, Argentina. Geobios, 39:553-560. doi:10.1016/j. geobios.2009.03.002

Casal, G.A. \& Ibiricu, L. 2010a. Materiales asignables a Epachthosaurus Powell, 1990 (Sauropoda, Titanosauria), de la Formación Bajo Barreal, Cretácico Superior, Chubut, Argentina. Revista Brasilera de Paleontología, 13:247-256. doi:10.4072/ rbp.2010.3.08

Casal, G.A.; Ibiricu, L.M.; Martínez, R.D.; Luna, M.; González Svoboda, J.E. \& Ivany, E. 2015b. El registro fósil de la Formación Lago Colhué Huapi (Coniaciano-Maastrichtiano), Grupo Chubut, Argentina. Ameghiniana, 52:210-211.

Casal, G.A.; Luna, M.; Martínez, R.D.; Lamanna, M.; Sciutto, J.C. \& Ivany, E. 2006. La fauna Campaniana-Maastrichtiana? de la Formación Bajo Barreal en el E-SE del Lago Colhué Huapi, Provincia de Chubut, Argentina. Ameghiniana, 40:61R.

Casal, G.A.; Martínez, R.D.; Ibiricu, L.M.; González Riga, B.J. \& Foix, N. 2013. Tafonomía del dinosaurio terópodo Aniksosaurus darwini, Formación Bajo Barreal, Cretácico Tardío de Patagonia (Argentina). Ameghiniana, 50:571-592. doi:10.5710/ AMGH.23.08.2013.617

Casal, G.A.; Martínez, R.D.; Luna, M.; Ivany, E. \& Ibiricu, L.M. 2010. Un nuevo titanosaurio articulado de la Formación Bajo Barreal, Cretácico Superior tardío del Sur de Chubut. Aspectos ambientales y tafonómicos. Ameghiniana, 47:6R.

Casal, G.A.; Martínez, R.D.; Luna, M.; Sciutto, J.C. \& Lamanna, M. 2007. Aeolosaurus colhuehuapensis sp. nov. (Sauropoda, Titanosauria) de la Formación Bajo Barreal, Cretácico Superior de Argentina. Revista Brasileira de Paleontologia, 10:53-62.

Casamiquela, R.M. 1964. Sobre un dinosaurio hadrosaurio de la Argentina. Ameghiniana, 3:285-308.

Carpenter, K.; Miles C. \& Cloward K. 2005. New small theropod from the Upper Jurassic Morrison Formation of Wyoming. In: K. Carpenter (ed.) The Carnivorous dinosaurs, Indiana University Press, p. 23-48.

Chatterjee, S. 1978. Indosuchus and Indosaurus, Cretaceous carnosaurs from India. Journal of Paleontology, 52:570-580.

Chelotti, L.A. \& Homovc, J.F. 1998. Estilos estructurales en la zona marginal de la Faja plegada de San Bernardo y su relación con los sistemas petroleros, Sarmiento, Chubut, Argentina. In: CONGRESO LATINOAMERICANO DE GEOLOGÍA ECONÓMICA, 10, 1998. Actas, Buenos Aires, p. 81-86. 
Clyde, W.C. 2014. New age constraints for the Salamanca Formation and lower Río Chico Group in the western San Jorge Basin, Patagonia, Argentina: Implications for Cretaceous-Paleogene extinction recovery and land mammal age correlations. Geological Society of America Bulletin, 126: 289-306. doi:10.1130/B30915.1

Coria, R.A. 1999. Ornithopod dinosaurs from the Neuquen Group, Patagonia, Argentina: phylogeny and biostratigraphy. In: Y. Tomid; T. Rich \& P. Vickers-Rich (eds.) Proceedings of Second Gondwanan Dinosaur Symposium, Tokyo, National Science Museum, p. 47-60 (Monographs 15).

Coria R.A. 2007. Nonavian Theropods. In: Z. Gasparini; L. Salgado \& R.A Coria (eds.) Patagonian Mesozoic Reptiles, Indiana University Press, p. 229-270.

Coria, R.A. 2010. Phylogeny and paleobiogeography of hadrosaurid dinosaurs from Argentina. In: CONGRESO LATINOAMERICANO DE PALEONTOLOGÍA, 7, 2010. Actas, La Plata, p. 149-150.

Coria, R.A. 2012. South American Hadrosaurs. The gentle geodispersal. In: INTERNATIONAL SYMPOSIUM ABOUT DINOSAURS, PALAEONTOLOGY AND THEIR ENVIRONMENT, 5, 2012. Proceedings, Burgos, p. 31-39.

Coria, R.A. \& Cambiaso, A. 2007. Ornithischian dinosaurs. In: Z. Gasparini; L. Salgado \& R.A. Coria (eds.) Patagonian Mesozoic Reptiles, Indiana University Press, p. 167-187.

Coria, R.A. \& Currie, P.J. 2006. A new carcharodontosaurid (Dinosauria, Theropoda) from the Upper Cretaceous of Argentina. Geodiversitas, 28:71-118.

Coria, R.A.; González Riga, B.J. \& Casadío, S. 2012. Un nuevo Hadrosáurido (Dinosauria, Ornithopoda) de la Formación Allen, provincia de La Pampa, Argentina. Ameghiniana, 49: 552-572. doi:10.5710/AMGH.9.4.2012.487

Coria, R.A. \& Salgado, L. 2005. Mid-Cretaceous turnover of saurischian dinosaur communities: evidence from the Neuquén Basin. In: G.D. Veiga; L.A. Spalletti, J.A. Howell \& E. Schwarz (eds.) The Neuquén Basin, Argentina: a case study in sequence stratigraphy and basin dynamics, London, Geological Society of London, p. 317-327. doi:10.1144/GSL.SP.2005.252.01.16

Currie, P.J. \& Chen, P.J. 2001. Anatomy of Sinosauropteryx primia from Liaoning, northeastern China. Canadian Journal of Earth Sciences, 38:1705-1727. doi:10.1139/e01-050

Currie, P.J.; Rigby, J.K. \& Sloan, R.E. 1990. Teropod teeth from the Judith River Formation of southern Alberta, Canada. In: K. Carpenter \& P.J. Currie (eds.) Dinosaur Systematics: perspectives and approaches, Cambridge University Press, $\mathrm{p}$. 107-125.

Currie, P.J. \& Zhao, X.J. 1993. A new carnosaur (Dinosauria: Theropoda) from the Jurassic of Xinjiang, People's Republic of China. Canadian Journal of Earth Sciences, 30:2037-2081. doi:10.1139/e93-179

Curry Rogers, K.A. 2005. Titanosauria: a phylogenetic overview. In: K.A. Curry Rogers \& J.A. Wilson (eds.) The Sauropods: evolution and paleobiology, University of California Press, p. 50-103. doi:10.1525/california/9780520246232.001.0001

De Klerk, W.J.; Forster, C.A.; Sampson, S.D.; Chinsamy, A. \& Ross, C.F. 2000. A new coelurosaurian dinosaur from the Early Cretaceous of South Africa. Journal of Vertebrate Paleontology, 20: 324-332. doi:10.1671/0272-4634(2000)020[0324:ANCDF T]2.0.CO;2

Depéret, C. 1896. Sur l'existence de dinosauriens, sauropodes et théropodes dans le Crétacé supérieur de Madagascar. Comptes Rendus de l'Académie des Sciences (Paris), Série II, 122:483-485.
Dodson, P. 1990. On the status of the ceratopsids Monoclonius and Centrosaurus. In: K. Carpenter \& P.J. Currie (eds.) Dinosaur Systematics: approaches and perspectives, Cambridge University Press, p. 231-243.

Dodson, P. \& Currie, P.J. 1988. The smallest ceratopsid skull, Judith River Formation of Alberta. Canadian Journal of Earth Sciences, 25:926-930. doi: 10.1139/e88-090

Ferello, R. \& Tealdi, O.L. 1950. Descripción del perfil general del flanco Oriental del Anticlinal de la Sierra del Castillo. Buenos Aires, Yacimientos Petrolíferos Fiscales.

Feruglio, E. 1949. Descripción geológica de la Patagonia.

Figari, E.G.; Strelkov, E.; Cid de La Paz, M.S.; Celaya, J.; Laffitte, G. \& Villar, H. 2002. Cuenca del Golfo San Jorge: síntesis estructural, estratigráfica y geoquímica. In: CONGRESO GEOLÓGICO ARGENTINO, 15, 2002. Relatorio, Buenos Aires, p. 571-601.

Figari, E.G.; Strelkov, E.; Laffitte, G.; Cid de La Paz, M.S.; Courtade, S.F.; Celaya, J.; Vottero, A.; Lafourcade, P.; Martínez, R. \& Villar, H.J. 1999. Los sistemas petroleros de la Cuenca del Golfo San Jorge: síntesis estructural, estratigráfica y geoquímica. In: CONGRESO DE EXPLORACIÓN Y DESARROLLO DE HIDROCARBUROS, 4, 1999. Actas, Mar del Plata, p. 197-237.

Gilmore, C.W. 1920. Osteology of the carnivorous Dinosauria in the United States National Museum, with special reference to the genera Antrodemus (Allosaurus) and Ceratosaurus. United States National Museum Bulletin, 110:1-154.

Gobbo-Rodrigues, S.R.; Petri, S. \& Bertini, R.J. 1999. Ocorrências de ostrácodes na Formação Adamantina do Grupo Bauru, Cretáceo Superior da Bacia do Paraná e possibilidades de correlação com depósitos isócronos argentinos. Parte I - Família Ilyocyprididae. Acta Geológica Leopoldensia, 23:3-13.

González Riga, B.J. 1995. Estratigrafía y Paleontología de vertebrados del Cretácico Superior de Ranquil-Có, sur de la provincia de Mendoza, Argentina. Programa de Postgrado, Facultad de Ciencias Exactas, Físicas y Naturales, Universidad Nacional de Córdoba, Tesis Doctoral, 133 p.

González Riga, B.J. 1999. Hallazgo de vertebrados fósiles en la Formación Loncoche, Cretácico Superior de la provincia de Mendoza, Argentina. Ameghiniana, 36:401-410.

González Riga, B.J. \& Casadío, S. 2000. Primer registro de Dinosauria (Ornithischia, Hadrosauridae) en la Provincia de La Pampa (Argentina) y sus implicancias paleobiogeográficas. Ameghiniana, 37:341-351.

Hechem, J.J., Homovc, J.F. \& Figari, E.G. 1990. Estratigrafía del Chubutiano (Cretácico) en la Sierra de San Bernardo, cuenca del Golfo San Jorge, Argentina. In: CONGRESO GEOLÓGICO ARGENTINO, 11, 1990. Actas, San Juan, Argentina, p. 173-176.

Herngreen, G.F.W.; Kedves, M.; Rovnina, L.V. \& Smirnova, S.B. 1996. Cretaceous palynofloral provinces: a review. In: J. Jansonius \& D.C. McGregor (eds.) Palynology: principles and applications. American Association of Stratigraphic Palynologists Foundation, p. 1157-1188.

Hooker, J.J.; Milner, A.C. \& Sequeira S.E.K. 1991. An ornithopod dinosaur from the Late Cretaceous of West Antarctica. Antarctic Science, 3:331-332. doi: 10.1017/S0954102091000391

Huene, F.F. von. 1929. Los saurischios y ornitisquios del Cretácico Argentino. Anales del Museo de La Plata, 3:1-194.

Huene, F. von. 1932. Die fossile Reptil-Ordnung Saurischia, ihre Entwicklung und Geschichte Teil I and II. Monographien zur Geologie und Palaeontologie, 1:1-361.

Ibiricu, L.M.; Casal, G.A.; Lamanna, M.; Martínez, R.; Harris. J. \& Lacovara. J. 2012a. The southernmost records of Rebbachisauridae (Sauropoda: Diplodocoidea), from the early 
Late Cretaceous of central Patagonia. Cretaceous Research, 34:220-232. doi: 10.1016/j.cretres.2011.11.003

Ibiricu, L.M.; Lamanna, M.C. \& Lacovara, K.J. 2014. The influence of caudofemoral musculature on the titanosaurian (Saurischia: Sauropoda) tail skeleton: morphological and phylogenetic implications: Historical Biology, 26:454-471. doi: 10.1080/08912963.2013.787069

Ibiricu, L.M.; Casal, G.A.; Martínez, R.D.; Lamanna, M.C.; Luna, M. \& Salgado, L. 2015. New material of Katepensaurus goicoecheai (Sauropoda: Diplodocoidea) and its significance for the morphology and evolution of Rebbachisauridae. Ameghiniana, 52:430-446. doi: 10.5710/AMGH.24.04.2015.2830

Ibiricu, L.; Casal, G.A.; Martínez, R. \& Navarrete, C. 2011. Materiales asignables a Titanosauria (Saurischia; Sauropoda) de la Formación Bajo Barreal, Cretácico Superior, Patagonian Central. Brazilian Geographical Journal, 2:337-353.

Ibiricu, L.M.; Casal G.A.; Martínez, R.D.; Lamanna, M.C. \& Salgado, L. 2013c. Katepensaurus goicocheai gen. et sp. nov., a Late Cretaceous rebbachisaurid (Sauropoda, Diplodocoidea) from central Patagonia, Argentina. Journal of Vertebrate Paleontology, 33:1351-1366. doi: 10.1080/02724634.2013.776562

Ibiricu, L.M.; Martínez, R.D. \& Casal, G.A. 2012b. The first record of Pterosauria in Bajo Barreal Formation (upper Cretaceous), central Patagonia, Argentina. Ameghiniana, 49:657-661. doi: 10.5710/AMGH.28.4.2011.520

Ibiricu, L.M.; Martínez, R.D.; Casal, G.A. \& Cerda, I.A. 2013b. The behavioural implications of a multi-individual bonebed of a small theropod dinosaur. PloS ONE, 8:e64253. doi: 10.1371/ journal.pone.0064253

Ibiricu, L.M.; Martínez, R.D.; Lamanna, M.C.; Casal, G.A.; Luna, M.; Harris, J. \& Lacovara, K.J. 2010. A medium sized Ornithopod (Dinosauria: Ornithischia) from the Upper Cretaceous Bajo Barreal Formation of Lago Colhué Huapi, Southern Chubut province, Argentina. Annals of the Carnegie Museum, 79:39-50. doi: 10.2992/007.079.0103

Ibiricu, L.M.; Martínez, R.D.; Luna, M. \& Casal, G.A. 2014. A reappraisal of Notohypshilophodon comodorensis (Ornithischia: Ornithopoda) from the Late Cretaceous of Patagonia, Argentina. Zootaxa, 3786:401-422. doi: 10.11646/zootaxa.3786.4.1

Juárez Valieri, R.D.; Haro, J.A.; Fiorelli, L.E. \& Calvo, J.O. 2010. A new hadrosauroid (Dinosauria: Ornithopoda) from the Allen Formation (Late Cretaceous) of Patagonia, Argentina. Revista del Museo Argentino de Ciencias Naturales, 12:217-231.

Krause, D.W.; Sampson, S.D.; Carrano, M.T. \& O’Connor, P.M. 2007. Overview of the history of discovery, Taxonomy, Phylogenia and Biogeography of Majungasaurus crenatissimus (Theropoda:Abelisauridae) from the Late Cretaceous of Madagascar. Journal of Vertebrate Paleontology, 27:1-20. doi: 10.1671/0272-4634(2007)27[1:OOTHOD]2.0.CO;2

Lamanna, M.; Casal, G. \& Martínez, R. 2012. A new Abelisaurid (Theropoda: ceratosauria) skeleton from the Upper Cretaceous Bajo Barreal Formation of Chubut Province, Argentina. Journal of Vertebrate Paleontology, 32:124.

Lamanna, M.C.; Luna, M.; Casal, G.A.; Martínez, R.D.; Ibiricu, L.M. \& Sciutto, J.C. 2003. New Crocodyliform and Dinosaur Discoveries From The Upper Cretaceous (CampanianMaastrichtian?) Upper Member Of The Bajo Barreal Formation, Southern Chubut Province, Argentina. Journal of Vertebrate Paleontology, 23:70A.

Lamanna, M.C.; Martínez, R.D.; Luna, M.; Casal, G.A.; Ivany, E. \& Ibiricu, L.M. 2004. New specimens of the problematic large theropod dinosaur Megaraptor from the Late Cretaceous of Central Patagonia. Journal of Vertebrate Paleontology, 24:81A.
Lamanna, M.C.; Martínez, R.D. \& Smith, J.B. 2002. A definitive abeli saurid theropod dinosaur from the Early Late Cretaceous of Patagonia. Journal of Vertebrate Paleontology, 22:58-69. doi: 0.1671/0272-4634(2002)022[0058:ADATDF]2.0.CO;2

Lapparent de Broin, F. \& de la Fuente, M.S. 2001. Oldest world Chelidae (Chelonii, Pleurodira), from the Cretaceous of Patagonia, Argentina. Comptes Rendues Académie des Sciences, 333:463-470. doi: 10.1016/S1251-8050(01)01662-7

Lavocat, R. 1954. Sur les dinosauriens du Continental Intercalaire des Kem Kem de la Daoura. Comptes Rendus, Alger, p. 65-68.

Leanza, H.; Apesteguía, S.; Novas, F.E. \& de la Fuente, M.S. 2004. Cretaceous terrestrial beds from the Neuquén Basin (Argentina) and their tetrapod assemblages. Cretaceous Research, 25:61-87. doi: 10.1016/j.cretres.2003.10.005

Lesta, P. \& Ferello, R. 1972. Región extraandina del Chubut y norte de Santa Cruz. In: A.F. Leanza (ed.) Geología Regional Argentina, Academia Nacional de Ciencias, p. 601-654.

Luna, M.; Casal, G.A.; Martínez, R.D.; Lamanna, M.C.; Ibiricu, L.M. \& Ivany, E. 2003. La presencia de un Ornitopoda en el Miembro Superior de La Formación Bajo Barreal, Cretácico Superior (Campaniano-Maastrichtiano?) del Sur de Chubut. Ameghiniana, p. 40:61R.

Lydekker, R. 1893. The Dinosaurs of Patagonia. Anales del Museo de La Plata, 2:1-14.

Mannion P.D. \& Calvo J.O. 2011. Anatomy of the basal titanosaur (Dinosauria, Sauropoda) Andesaurus delgadoi from the midCretaceous (Albian-early Cenomanian) Rio Limay Formation, Neuquen Province, Argentina: implications for titanosaur systematics. Zoological Journal of the Linnean Society, 163:155181. doi: 10.1111/j.1096-3642.2011.00699.x

Mannion, P.D. \& Otero, A. 2012. A reappraisal of the Late Cretaceous Argentinean saurópodo dinosaur Argyrosaurus superbus, with a description of a new titanosaur genus. Journal of Vertebrate Paleontology, 32:614-638. doi: 10.1080/02724634.2012.660898

Martinelli, A.G.; Riff, D. \& Lopes R.P. 2011. Discussion about the occurrence of the genus Aeolosaurus Powell 1987 (Dinosauria, Titanosauria) in the Upper Cretaceous of Brazil. Gaea, 7:34-40. doi: 10.4013/gaea.2011.71.03

Martínez, R.D. 1998a. An articulated skull and neck of Sauropoda (Dinosauria: Saurischia) from the Upper Cretaceous of Central Patagonia, Argentina. Journal of Vertebrate Paleontology, 18:61A.

Martínez, R.D. 1998b. Notohypsilophodon comodorensis gen. sp. nov., un Hypsilophodontidae (Ornithischia: Ornithopoda) del Cretácico Superior de Chubut, Patagonia Central, Argentina. Acta Geológica Leopoldensia, 21:119-135.

Martínez, R.D. 2007. Los dinosaurios de la Fm. Bajo Barreal: Testimonios de un mundo desaparecido. In: JORNADAS DE ARQUEOLOGÍA Y PALEONTOLOGÍA, 1, 2007. Actas, Pico Truncado, p. 88-104.

Martínez, R.D.; Casal, G.A. \& Luna, M. 2008. Los Dinosaurios del Chubut. Rawson, Secretaría de Cultura de la Provincia del Chubut, $136 \mathrm{p}$.

Martínez, R.D.; Giménez, O.; Rodríguez, J. \& Bochatey, G. 1986. Xenotarsosaurus bonapartei gen. sp. nov. (CarnosauriaAbelisauridae), un nuevo Theropoda de la Fm. Bajo Barreal, Chubut, Argentina. In: CONGRESO ARGENTINO DE PALEONTOLOGÍA Y BIOESTRATIGRAFÍA, 4, 1986. Actas, Mendoza, p. 23-31.

Martínez, R.D.; Giménez, O.; Rodríguez, J. \& Luna, M. 1990. Un titanosáurido articulado del género Epachthosaurus, de la Formación Bajo Barreal, Cretácico Superior del Chubut. Ameghiniana, 26:246. 
Martínez, R.D.; Giménez, O.; Rodríguez, J.; Luna, M. \& Lamanna, M. 2004a. An articulated specimen of the basal Titanosaurian (Dinosauria: Saurópoda) Epachthosaurus sciuttoi from the Early Late Cretaceous Bajo Barreal Formation of Chubut Province, Argentina. Journal of Vertebrate Paleontology, 24:107-120. doi: 10.1671/9.1

Martínez, R.D.; Lamanna, M.C.; Casal, G.A.; Luna, M.; Dodson, P.; Tiedemann, C. \& Koprowski, A. 2001. Dinosaurios de la Formación Bajo Barreal, Cretácico Superior Temprano del Sur del Chubut. Ameghiniana, 38:12R.

Martínez, R.; Lamanna, M.C.; Casal, G.A.; Luna, M.; Ivany, E.; Mansilla, V. \& Krause, M. 2005. Nuevos restos de Theropoda del Cretácico Superior temprano de la Patagonia central. In: JORNADAS ARGENTINAS DE PALEONTOLOGÍA DE VERTEBRADOS, 21, 2005. Resúmenes, Plaza Huincul, p.74R.

Martínez, R.D.; Lamanna, M.C.; Novas, F.E.; Ridgely, R.C.; Casal, G.A.; Martínez, J.E.; Vita, J.R. \& Witmer, L.M. 2016. A basal lithostrotian titanosaur (Dinosauria: Sauropoda) with a complete skull: implications for the evolution and paleobiology of Titanosauria. PLos ONE, 11:e0151661. doi:10.1371/journal. pone. 0151661

Martínez, R.D.; Maure, A.; Oliva, M. \& Luna, M. 1993.Un maxilar de Theropoda (Abelisauria) de la Formación Bajo Barreal, Cretácico tardío, Chubut, Argentina. Ameghiniana, 30:109-110.

Martínez, R.D. \& Novas, F. 2006. Aniksosaurus darwini gen. et sp. nov., a new coelurosaurian theropod from the Early Late Cretaceous of Central Patagonia, Argentina. Revista del Museo Argentino de Ciencias Naturales, 8:243-259.

Martínez, R.D.; Novas, F.E. \& Ambrosio, A. 2004b. Abelisaurid remains (Theropoda, Ceratosauria) from Southern Patagonia. Ameghiniana, 41:577-585.

Martínez, R.D.; Vita, J.; Lamanna, M.C. \& Ibiricu, L.M. 2006. A CT scan of a titanosauriform skull (Dinosauria: Sauropoda) from Central Patagonia, Argentina. Journal of Vertebrate Paleontology, 26:96A.

Martínez, R.D.; Vita, J.; Navarrete, C.; Casal, G.A. \& Lamanna, M.C. 2009. Tomografía helicoidal computada de vértebras cervicales de un Titanosauriforme (Dinosauria: Sauropoda) del sur del Chubut. Ameghiniana, 46:36-37R.

Miall, A.D. 1996. The geology of fluvial deposits: sedimentary facies, basin analysis and petroleum geology. Berlin, SpringerVerlag, $582 \mathrm{p}$.

Navarrete, C.; Casal, G.A. \& Martínez, R.D. 2011. Drusilasaura deseadensis gen. et sp. nov., un nuevo titanosaurio (DinosauriaSauropoda) de la Formación Bajo Barreal, Cretácico Superior del Norte de Santa Cruz, Argentina. Revista Brasileira de Paleontología, 14:1-14. doi: 10.4072/rbp.2011.1.01

Norman, D.B.; Hans-Dieter, S.; Witmer, M. \& Coria, R.A. 2004. Basal Ornithopoda. In: D.B. Weishampel; P. Dodson \& H. Osmolska (eds.) The Dinosauria, University of California Press, p. 393-412.

Novas, F.E. 1997. South American dinosaurs. In: K. Padian \& P. Currie (eds.) Encyclopedia of dinosaur, Academic Press, p. 678-689.

Novas, F.E. 1998. Megaraptor namunhuaiquii, gen. et sp. a large clawed, Late Cretaceous theropod from Patagonia. Journal of Vertebrate Paleontology, 18:4-9. doi: 10.1080/02724634.1998.10011030

Novas, F.E. 2009. The Age of Dinosaurs in South America. Bloomington, Indiana University Press, $452 \mathrm{p}$.

Novas, F.E.; de Valais, S.; Vickers-Rich, P. \& Rich, T. 2005. A large Cretaceous theropod from Patagonia, Argentina, and the evolution of carchadorontosaurids. Natturwissenschaften, 92:226-230.
Novas, F.E.; Ezcurra, M.D.; Agnolin, F.L.; Pol, D. \& Ortiz, R. 2012. New Patagonian Cretaceous theropod sheds light about the early radiation of Coelurosauria. Revista Museo Argentino de Ciencias Naturales, 14:57-81.

Osborn, H.F. 1903. Ornitholestes hermanni, a new compsognathid dinosaur from the Upper Jurassic. Bulletin of the American Museum of Natural History, 19:459-464.

Paredes, J.M. 2009. Sedimentary evolution of the Golfo San Jorge basin, central Patagonia, Argentina. In: L.M. Ibañez; S. Moyano \& G. Aceñolaza (eds.) Argentinean fluvial basins: ancient and present day examples. Instituto Miguel Lillo, p. 187-275.

Poropat, S.F.; Upchurch, P.; Mannion, P.D.; Hockull, S.A.; Kear, B.P.; Sloan, T.; Sinapius, H.K. \& Elliott, D.A. 2015. Revision of the sauropod dinosaur Diamantinasaurus matildae Hocknull et al., 2009 from the mid- Cretaceous of Australia: implications for Gondwanan titanosauriform dispersal. Gondwana Research, 27:995-1033. doi: 10.1016/j.gr.2014.03.014

Powell, J.E. 1987. The Late Cretaceous fauna from Los Alamitos, Patagonia, Argentina. The titanosaurids. Revista Museo Argentino Ciencias Naturales, 3:147-153.

Powell, J.E. 1990. Epachthosaurus sciuttoi (gen. et sp. nov.) un dinosaurio saurópodo del Cretácico de Patagonia (Provincia de Chubut, Argentina). In: CONGRESO ARGENTINO DE PALEONTOLOGÍA Y BIOESTRATIGRAFÍA, 4, 1990. Actas, Tucumán, p. 23-128.

Powell, J.E. 2003. Revision of South American titanosaurid dinosaurs: palaeobiological, palaeobiogeographical, and phylogenetic aspects. Records of the Queen Victoria Museum, 111:1-173.

Powell J.; Giménez, O.; Martínez, R. \& Rodríguez, J. 1989. Nuevo hallazgo de saurópodos en la Formación Bajo Barreal de "Ocho Hermanos", Sierra de San Bernardo, provincia del Chubut, y su significado cronológico. In: CONGRESSO BRASILEIRO DE PALEONTOLOGIA, 11, 1989. Anais, Curitiba, SBP, p. 165-176.

Previtera, E. \& González Riga, B.J. 2008. Vertebrados cretácicos de la Formación Loncoche en Calmu-Co, Mendoza, Argentina. Ameghiniana, 45:349-359.

Prieto-Marquez, A. 2010. Global historical biogeography of hadrosaurid dinosaurs. Zoological Journal of the Linnean Society, 159:503-525. doi:10.1111/j.1096-3642.2010.00642.x

Prieto-Marquez, A. \& Salinas, G.C. 2010. A re-evaluation of Secernosaurus koerneri and Kritosaurus australis (Dinosauria, Hadrosauridae) from the Late Cretaceous of Argentina. Journal of Vertebrate Paleontology, 30:813-837. doi: 10.1080/02724631003763508

Pujana, R.; Umazano, A. \& Bellosi, E. 2007. Maderas fósiles afines a Araucariaceae de la Formación Bajo Barreal, Cretácico Tardío de Patagonia central (Argentina). Revista del Museo Argentino de Ciencias Naturales, 9:161-167.

Retallack, G.J. 1988. Field recognition of paleosoils. In: J. Reinhardt \& W.R. Sigleo (eds.) Paleosols and weathering through geologic time: principles and applications, Boulder, Geological Society of America, p.1-20 (Special Paper 216).

Rodríguez, J.F.R. 1992. Interpretación paleoambiental de la Formación Bajo Barreal (Cretácico Tardío) en Estancia Ocho Hermanos, Chubut. In: REUNIÓN DE SEDIMENTLOGÍA, 4, 1992. Actas, La Plata, p. 81-88.

Rodríguez, J.F.R. 1993. La depositación de las areniscas verdes (Formación Bajo Barreal, Cretácico Tardío) y sus implicancias taxonómicas. In: CONGRESO GEOLÓGICO, 12, CONGRESO DE EXPLORACIÓN DE HIDROCARBUROS, 3, 1993. Actas, Mendoza, p. 194-199. 
Roll, A. 1938. Estudio geológico de la zona sur del curso medio del río Deseado. Boletín de Informaciones Petroleras, 163:69-88.

Salgado, L. \& Bonaparte, J.F. 2007. Sauropodomorpha. In: Z. Gasparini; L. Salgado \& R.A. Coria (eds.) Patagonian mesozoic reptiles, Indiana University Press, p. 188-228.

Salgado, L. \& Coria, R.A. 1993. El género Aeolosaurus (Sauropoda. Titanosauridae) en la Formación Allen (CampanianoMaastrichtiano) de la Provincia de Río Negro, Argentina. Ameghiniana, 30:119-128.

Salgado, L. \& Coria, R.A. 1996. First evidence of an ankylosaur (Dinosauria, Ornithischia) in South America. Ameghiniana, 33:367-371.

Salgado, L.; Coria, R.A. \& Calvo, J.O. 1997a. Evolution of titanosaurid sauropods I: phylogenetic analysis based on the postcranial evidence. Ameghiniana, 34:3-32.

Salgado, L.; Coria, R.A \& Calvo, J.O. 1997b. Presencia del género Aeolosaurus (Sauropoda, Titanosauridae) en la Formación Los Alamitos, Cretácico Superior de la Provincia de Río Negro, Argentina. Revista Universidade Guarulhos, 2:44-49.

Salgado,L.; Garrido,A.; Cocca, S. \& Cocca, J.R. 2004.LowerCretaceous rebbachisaurid sauropods from Cerro Aguada del León (Lohan Cura Formation), Neuquén Province, northwestern Patagonia, Argentina. Journal of Vertebrate Paleontology, 24:903-912. doi: 10.1671/0272-4634(2004)024[0903:LCRSFC]2.0.CO;2

Salgado, L. \& Martínez, R.D. 1993. Relaciones filogenéticas de los titanosáuridos basales Andesaurus delgadoi y Epachthosaurus sp. Ameghiniana, 30:339.

Sankey, J.; Brinkman, D.; Guenther, M. \& Currie, P. 2002. Small theropod and bird theet from the Late Cretaceous (Late Campanian) Judith River Group, Alberta. Journal of Vertebrate Paleontology, 76:751-763. doi: 10.1666/0022-3360(2002)076 $<0751$ :STABTF>2.0.CO;2

Santucci, R.M. \& de Arruda-Campos, A.C. 2011. A new sauropod (Macronaria, Titanosauria) from the Adamantina Formation, Bauru Group, Upper Cretaceous of Brazil and the phylogenetic relationships of Aeolosaurini. Zootaxa, 3085:1-33.

Sampson, S.D.; Witmer, L.M.; Forster, C.A.; Krause, D.W.; O'Connor, P.M.; Dodson, P. \& Ravoavy, F. 1998. Predatory dinosaur remains from Madagascar: implications for the Cretaceous biogeography of Gondwana. Science, 280:10481051. doi: $10.1126 /$ science.280.5366.1048

Sciutto, J.C. 1981. Geología del codo del Río Senguerr, Chubut, Argentina. In: CONGRESO GEOLÓGICO ARGENTINO, 8, 1981. Actas, San Luis, p. 203-219.

Sciutto, J.C.; Césari, O. \& Iantanos, N. 2008. Hoja geológica 4569IV Escalante, escala 1:250000: Programa Nacional de Cartas Geológicas de la República Argentina. Buenos Aires, Secretaría de Minería de la Nación, Dirección Nacional del Servicio Geológico. (Boletín 351).

Sciutto, J.C. \& Martínez, R.D. 1994. Un nuevo yacimiento fosilífero de la Formación Bajo Barreal (Cretácico tardío) y su fauna de saurópodos. Naturalia Patagónica, Ciencias de la Tierra, 2:27-47.

Sereno, P.; Wilson, J. \& Conrad, J. 2004. New dinosaurs link southern landmasses in the Mid-Cretaceous. Proceedings of the Royal Society of London B: Biological Sciences, 271:1325-1330. doi: 10.1098/rspb.2004.2692
Simeoni, M. 2014. Nannofossils and foraminifera from the Salamanca Formation (Paleocene) in Punta Peligro Norte (Chubut, Argentina). Carnets de Géologie Notebooks on Geology, 14:343-349.

Spalletti, L.A. \& Franzese, J.R. 2007. Mesozoic Paleogeography and Paleoenvironmental Evolution of Patagonia (Southern South America). In: Z. Gasparini; L. Salgado \& R.A. Coria (eds.) Patagonian mesozoic reptiles, Indiana University Press, p. 29-49.

Suárez, M.; Márquez, M.; De La Cruz, R.; Navarrete, C. \& Fanning, M. 2014. Cenomanian-? early Turonian minimum age of the Chubut Group, Argentina: SHRIMP U-Pb geochronology. Journal of South American Earth Sciences, 50:67-74. doi: 10.1016/j.jsames.2013.10.008

Tapia, A. 1919. Una mandibula de Dinosaurio procedente de Patagonia. Revista de la Sociedad Argentina de Ciencias Naturales, 4:369-370.

Teruggi, M.E. \& Rossetto, H. 1963. Petrología del Chubutiano del codo del río Senguerr. Boletín de Informaciones Petroleras, 354:18-35.

Uliana, M. \& Legarreta, L. 1999. Jurásico y Cretácico de la Cuenca del Golfo San Jorge. In: R. Caminos (ed.) Geología Argentina, Buenos Aires, Subsecretaría de Minería de la Nación, Instituto de Geología y Recursos Minerales, p. 496-510 (Anales 29).

Umazano, A.M.; Bellosi, E.S.; Visconti, G. \& Melchor, R.N. 2008. Mechanisms of aggradation in fluvial systems influenced by explosive volcanism: an example from the Late Cretaceous Bajo Barreal Formation, San Jorge Basin, Argentina. Sedimentary Geology, 203:213-228. doi: 10.1016/j.sedgeo.2007.12.001

Umazano, A.M.; Bellosi, E.S.; Visconti, G. \& Melchor, R.N. 2012. Detecting allocyclic signals in volcaniclastic fluvial successions: Facies, architecture and stacking pattern from the Cretaceous of central Patagonia, Argentina. Journal of South American Earth Sciences, 40:94-115. doi: 10.1016/j.jsames.2012.09.005

Vallati, P.; Casal, G.; Foix, N.; Allard, J.; de Sosa Tomas, A. \& Calo, M. 2016. First report of a Maastrichtian palynoflora from the Golfo San Jorge Basin, central Patagonia, Argentina. Ameghiniana, in press. doi: 10.5710/AMGH.28.12.2015.2948

Vilela, C. 1971. Descripción geológica de la Hoja 48c, "Lago Musters”, provincia del Chubut, Carta Geológico-Económica de la República Argentina, escala 1:200.000. Buenos Aires, Ministerio de Industria y Minería, 63p. (Boletín 113).

Weishampel, D.B. \& Horner, J. 1990. Hadrosauridae. In: D.B. Weishampel; P. Dodson \& H. Osmólska (eds.) The Dinosauria, University of California Press, p. 534-561.

Windhausen, A. 1918. The problem of de Cretaceous-Tertiary boundary in South America and stratigraphic position of the San Jorge Formation in Patagonia. American Journal of Science, 45:2-53.

Whitlock, J.A. 2011. A phylogenetic analysis of Diplodocoidea (Saurischia: Sauropoda). Zoological Journal of the Linnean Society, 161:872-915. doi: 10.1111/j.1096-3642.2010.00665.x

Zaaboub, N.; Abdeljaouad, S. \& López-Galindo, A. 2005. Origin of fibrous clays in Tunisian Paleogene continental deposits. Journal of African Earth Sciences, 43:491-504. doi:10.1016/j. jafrearsci.2005.08.013

Received in October, 2015; accepted in February, 2016. 